\title{
Stable mixed finite elements for linear elasticity with thin inclusions
}

\author{
W. M. Boon ${ }^{1,2}$ (D) J. M. Nordbotten ${ }^{3}$ \\ Received: 23 May 2019 / Accepted: 16 October 2020 / Published online: 6 November 2020 \\ (C) The Author(s) 2020
}

\begin{abstract}
We consider mechanics of composite materials in which thin inclusions are modeled by lower-dimensional manifolds. By successively applying the dimensional reduction to junctions and intersections within the material, a geometry of hierarchically connected manifolds is formed which we refer to as mixed-dimensional. The governing equations with respect to linear elasticity are then defined on this mixed-dimensional geometry. The resulting system of partial differential equations is also referred to as mixed-dimensional, since functions defined on domains of multiple dimensionalities are considered in a fully coupled manner. With the use of a semi-discrete differential operator, we obtain the variational formulation of this system in terms of both displacements and stresses. The system is then analyzed and shown to be well-posed with respect to appropriately weighted norms. Numerical discretization schemes are proposed using well-known mixed finite elements in all dimensions. The schemes conserve linear momentum locally while relaxing the symmetry condition on the stress tensor. Stability and convergence are shown using a priori error estimates and confirmed numerically.
\end{abstract}

Keywords Mixed finite element $\cdot$ Linear elasticity $\cdot$ Mixed-dimensional $\cdot$ Weak symmetry $\cdot$ A priori analysis

Mathematics Subject Classification (2010) $65 \mathrm{~N} 12 \cdot 65 \mathrm{~N} 30 \cdot 74 \mathrm{~S} 05 \cdot 74 \mathrm{~K} 20$

\section{Introduction}

Thin inclusions in elastic materials arise in a variety of scientific fields, including geo-physics, bio-mechanics, and the study of composite materials. The subsurface, for example, typically includes rock layers with significantly larger horizontal extent compared to their height. Since it is often infeasible to resolve such small heights for largescale simulations, we consider the setting where the layer, or aquifer, is represented by a lower-dimensional manifold. The governing equations on this manifold can be derived using vertical integration [22]; see e.g. [7] for an application regarding $\mathrm{CO}_{2}$ storage.

W. M. Boon

wietse@kth.se

1 Institute for Modelling Hydraulic and Environmental Systems, University of Stuttgart, Stuttgart, 70569, Germany

2 Present address: Department of Mathematics, KTH Royal Institute of Technology, Stockholm, 11428, Sweden

3 Department of Mathematics, University of Bergen, Bergen, 5020, Norway
Secondly, membranes occur frequently in the study of bio-mechanical systems. Examples range from cell walls in plants to the heart sac and dermal layer in human physiology. As a modeling assumption, each of these membranes can be represented by lower-dimensional manifolds. Their influence on the coupled mechanical system can then be incorporated by assigning significantly different material properties compared to the surroundings.

A third application concerns the study of composite or reinforced materials. In this context, the lower-dimensional manifolds correspond to the stiffer plates embedded in the material for strengthening purposes. This can be expanded to connected, two-dimensional objects such as H-beams and T-beams. The junctions are then considered onedimensional manifolds, with inherited or separately defined material properties. We note that this work is limited to manifolds of codimension one and thus does not treat the case of embedded, one-dimensional rods in three dimensions.

The thin features are considered lower-dimensional and have elastic properties, yet a slightly different setting is presented than the conventional theory of thin shells [13]. The main difference is that we focus on a strong coupling of a thin inclusion with a surrounding, elastic medium. The 
interest of this work is therefore more closely aligned to [12], in which rigid, thin inclusions are considered.

The structure of the derived equations fits well with the mixed-dimensional framework derived in $[9,21]$. We aim to preserve this structure and retain a local conservation of linear momentum after discretization with the use of conforming, mixed finite elements. The construction of stable finite element pairs representing displacements and symmetric stresses is involved, and typically leads to higherorder elements for the stress space [4]. By relaxing the symmetry condition on the stress tensor as in $[2,5]$, these difficulties can be mitigated.

While our derivation is different, our mixed-dimensional equations coincide in the case of a single thin inclusion with the elastic equations derived in [7]. In that work, significant effort was placed into model verification, including comparison to fixed-dimensional models. Their finding justified that the mixed-dimensional approach has sufficient accuracy for relevant physical problems such as $\mathrm{CO}_{2}$ storage, and allows for significant computational gains when coupled to fluid flow simulations (multiphase Biot). Further calculations are reported in the thesis [6]. More generally, mixed-dimensional models for high-aspect inclusions are well established and verified in the simpler case of scalar elliptic equations [19].

This article is structured as follows. Section 2 introduces the notational conventions and the decomposition of the geometry according to dimension. On this geometry, Section 3 introduces the governing equations of the model in all dimensions. We introduce the relevant function spaces and present the derivation of the variational formulation in Section 4. The resulting system of equations is proven to be well-posed in Section 5. Conforming discretization schemes are proposed in Section 6 for which stability and convergence are shown. Finally, Section 7 presents twodimensional numerical experiments to verify the theoretical results.

\section{Geometry and notation}

In this section, we introduce the mixed-dimensional geometry and establish notation. Here, we follow the conventions introduced in $[9,10]$.

Let us consider an $n$-dimensional domain $Y$ that contains thin, embedded structures, represented by lowerdimensional manifolds. In general, we consider $n=3$, the two-dimensional case being simpler. Let $\Omega_{i}^{d_{i}}$ be such a manifold, with $i$ the unique index from a global set $I$ and $d_{i}$ its dimension. The superscript is frequently omitted for brevity. For $1 \leq d \leq n-1$, we successively identify intersections between $d$-manifolds as $(d-1)$-manifolds. All $\Omega_{i}$ are open sets and mutually disjoint. For simplicity, we restrict this work to the case in which all $\Omega_{i}$ have zero curvature, i.e., are flat.

As an example, let us consider the two-dimensional setup in Fig. 1 (left). Here, the cross-section of an embedded Hbeam is described using two zero-dimensional intersection points and five one-dimensional line segments. The open set corresponding to the surrounding medium is given by $\Omega_{8}^{2}=\operatorname{int}\left(Y \backslash \cup_{i=1}^{7} \Omega_{i}^{d_{i}}\right)$.

We refer to the union $\bigcup_{i \in I} \Omega_{i}$ as the mixed-dimensional geometry $\Omega$. Let $I^{d}$ be the set of indices corresponding to $d$-manifolds and let $\Omega^{d}$ be the collection of such manifolds. In short, we denote:

$I^{d}=\left\{i \in I: d_{i}=d\right\}, \quad \Omega^{d}=\bigcup_{i \in I^{d}} \Omega_{i}, \quad \Omega=\bigcup_{d=0}^{n} \Omega^{d}$.

The interface between manifolds of codimension one will play an important role, and we adopt a separate notation for these. Let $J$ be the set of indices such that each $j \in J$ corresponds to an interface $\Gamma_{j}$ between $\Omega_{i}$ for some $i \in I$ and an adjacent domain of dimension $\left(d_{i}+1\right)$. By definition, $\Gamma_{j}$ physically coincides with $\Omega_{i}$ and a unique $\hat{\jmath} \in I$ exists such that $\Gamma_{j} \subseteq \partial \Omega_{\hat{\jmath}}$.

To distinguish adjacent interfaces, we define the following index sets for $i \in I$ :

$\hat{J}_{i}=\left\{j \in J: \Gamma_{j}=\Omega_{i}\right\} \quad \check{J}_{i}=\left\{j \in J: \Gamma_{j} \subseteq \partial \Omega_{i}\right\}$.

An example is shown in Fig. 1 (right), which emphasizes that for $j_{1}, j_{2} \in \hat{J}_{4}$ with $j_{1} \neq j_{2}$, we have $\hat{\jmath}_{1}=\hat{\jmath}_{2}=8$. In other words, we allow for a manifold $\Omega_{\hat{\jmath}}$ to border on multiple sides of $\Omega_{i}$ and assign a unique index $j \in \hat{J}_{i}$ to each side. Finally, we remark that $\hat{J}_{i}$ is void for all $i \in I^{n}$, by definition.

Using the same summation convention per dimension as above, we denote:

$\Gamma^{d}=\bigcup_{i \in I^{d}} \bigcup_{j \in \hat{J}_{i}} \Gamma_{j}, \quad \Gamma=\bigcup_{d=0}^{n-1} \Gamma^{d}$.

Each $\Gamma_{j}$ is equipped with a unit normal vector $\boldsymbol{n}_{j}$, from the tangent space of $\Omega_{\hat{\jmath}}$, oriented outward with respect to $\Omega_{\hat{\jmath}}$. The subscript on $\boldsymbol{n}$ is omitted for brevity.

The boundary of the domain is given by the disjoint union $\partial_{\sigma} Y \cup \partial_{u} Y$ on which different boundary conditions will be imposed. In particular, we assume that the displacement is given on $\partial_{u} Y$ and the normal stress on $\partial_{\sigma} Y$. We denote for $i \in I$,

$\partial_{u} \Omega_{i}=\partial_{u} Y \cap \partial \Omega_{i}$,

$\partial_{\sigma} \Omega_{i}=\partial \Omega_{i} \backslash\left(\cup_{j \in \breve{J}_{i}} \Gamma_{j} \cup \partial_{u} \Omega_{i}\right)$.

For analysis purposes, we assume that $\left|\partial_{u} \Omega_{i}\right|>0$ for all $i \in I^{n}$, i.e., each subdomain of dimension $n$ is connected 


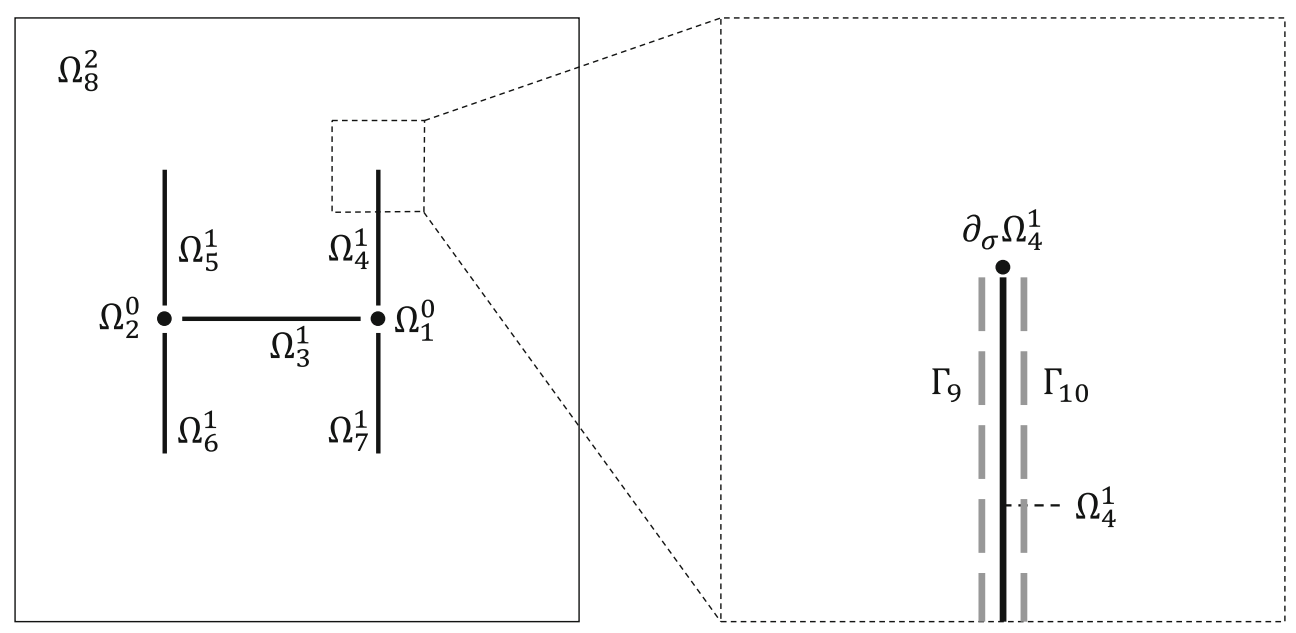

Fig. 1 The domain is decomposed into manifolds of different dimensionalities $\Omega_{i}^{d}$ with $i$ the global index and $d=d_{i}$ its dimension. (Left) The intersection points become zero-dimensional manifold in the decomposition. Here, the index sets are given by $I^{0}=\{1,2\}$, $I^{1}=\{3,4,5,6,7\}$, and $I^{2}=\{8\}$. (Right) A zoom on one of the

to a part of the boundary on which the displacement is prescribed. By omission of the subscript, we use $\partial_{u} \Omega$ and $\partial_{\sigma} \Omega$ to refer to the corresponding boundaries of the mixed-dimensional geometry.

Given a function $f$ defined on the mixed-dimensional geometry $\Omega$, let $f_{i}$ denote its restriction to $\Omega_{i}$, i.e. $f_{i}=$ ( $f)\left.\right|_{\Omega_{i}}$. Furthermore, we employ the hat and check notation to distinguish instances of $f$ inherited from different domains onto the interface $\Gamma$. This means that on $\Gamma_{j}$ with $j \in \hat{J}_{i}$, we denote:

$\check{f}:=f_{i}, \quad \hat{f}:=\left.\left(f_{\hat{\jmath}}\right)\right|_{\Gamma_{j}}$.

Note that the definition of $\hat{f}$ involves a trace of $f_{\hat{\jmath}}$ onto $\Gamma_{j}$.

\section{Model formulation}

In this section, we consider the governing equations and introduce the model problem. Starting with the mathematical formulation of linear elasticity in the surrounding medium, we continue with the generalized equations on lower-dimensional manifolds to derive the strong form of the mechanics problem. The variational formulation is considered afterward in Section 4.

\subsection{Governing equations in the surrounding medium}

Let us start by presenting the governing equations for linear elasticity in the surrounding medium $\Omega^{n}$. For $i \in I^{n}$, let $\sigma_{i}$ denote the elastic stress and $\boldsymbol{u}_{i}$ the displacement. Assuming extremities showing the logical interpretation of $\Omega_{4}^{1}$ and its interfaces $\left(\Gamma_{j}\right)$ with $\Omega_{8}^{2}$. In this case, we have $\hat{\jmath}=8$ for all $j \in \hat{J}_{4}=\{9,10\}$ and for $j_{1}, j_{2}, j_{3} \in \hat{J}_{1}$, we have $\left(\hat{\jmath}_{1}, \hat{\jmath}_{2}, \hat{\jmath}_{3}\right)=(3,4,7)$. On the extremity $\partial_{\sigma} \Omega_{4}^{1}$, a zero stress condition is imposed

infinitesimal strain, the stress-strain relationship has the general form:

$$
A \sigma_{i}=\varepsilon\left(\boldsymbol{u}_{i}\right)=\operatorname{sym}\left(\nabla_{i} \boldsymbol{u}_{i}\right)=\frac{1}{2}\left(\nabla_{i} \boldsymbol{u}_{i}+\left(\nabla_{i} \boldsymbol{u}_{i}\right)^{T}\right) .
$$

Here, the operator $\nabla_{i}$ is the standard del operator in $n$ dimensions. The subscript $i$ is there to ease the transition to the mixed-dimensional setting considered in later sections. In case of homogeneous and isotropic media, the operator $A$ describes Hooke's law and is given by:

$A \sigma_{i}=\frac{1}{2 \mu}\left(\sigma_{i}-\frac{\lambda}{2 \mu+n \lambda} \operatorname{Tr} \sigma_{i} I\right)$,

in which $\lambda$ and $\mu$ are the Lamé parameters and $\operatorname{Tr}$ is the matrix trace operator.

In the variational formulation presented in Section 4, the symmetry of the stress tensor $\sigma_{i}$ is enforced in a weak sense, which allows us to use low-order mixed finite element spaces. In preparation, we introduce the antisymmetric tensor variable $\chi_{i} \in \mathbb{R}_{\mathrm{asym}}^{n \times n}$ as:

$\chi_{i}:=\operatorname{asym} \nabla_{i} \boldsymbol{u}_{i}=\frac{1}{2}\left(\nabla_{i} \boldsymbol{u}_{i}-\left(\nabla_{i} \boldsymbol{u}_{i}\right)^{T}\right)$,

describing rotational motions. In turn, we have:

$A \sigma_{i}=\nabla_{i} \boldsymbol{u}_{i}-\chi_{i}$

With the addition of linear and angular momentum conservation, the following system of equations is formed in each $\Omega_{i}$ with $i \in I^{n}$.

$$
\begin{aligned}
A \sigma_{i}-\nabla_{i} \boldsymbol{u}_{i}+\chi_{i} & =0, \\
\nabla_{i} \cdot \sigma_{i} & =-\boldsymbol{f}_{i}, \\
\operatorname{asym} \sigma_{i} & =0 .
\end{aligned}
$$


With $f_{i}$ the body forces acting on $\Omega_{i}$. Note that the balance of angular momentum is enforced as the symmetry of the stress tensor $\sigma_{i}$ in Eq. 3.2c. The associated boundary conditions are given by:

$\boldsymbol{n} \cdot \sigma_{i}=0$ on $\partial_{\sigma} \Omega_{i}, \quad \boldsymbol{u}_{i}=\boldsymbol{g}_{u}$ on $\partial_{u} \Omega_{i}$.

with $\boldsymbol{g}_{u}$ a given function. We limit the exposition to homogeneous stress boundary conditions, noting that this can readily be extended to the general case.

For each $j \in \breve{J}_{i}$, the boundary condition on $\Gamma_{j}$ is considered a coupling condition and will therefore be presented in Section 3.3.

\subsection{Mixed-dimensional variables}

We devote this section to defining the relevant variables in the lower-dimensional domains. We introduce the stress and displacement on these manifolds as generalizations of $\sigma_{i}$ and $\boldsymbol{u}_{i}$ from the previous section, and we therefore let these inherit the respective notation.

Let us first define the stress variable. In $\Omega_{i}$ with $d_{i}<n$, let $\sigma_{i}$ be given by the columns of the Cauchy stress tensor associated with the tangent bundle of $\Omega_{i}$, integrated over the cross-section of the physical feature. As an example, for $i \in I^{n-1}$, let $Y_{i}$ be the physical $n$-dimensional feature before its reduction to the $(n-1)$-manifold $\Omega_{i}$. Letting $\tilde{\sigma}_{i}$ be the Cauchy stress tensor in $Y_{i}$, we define:

$\sigma_{i}\left(x_{\|}\right):=\int_{-\epsilon}^{\epsilon} \tilde{\sigma}_{i}\left(x_{\|}, x_{\perp}\right) I_{\|} \mathrm{d} x_{\perp}, \quad \forall x_{\|} \in \Omega_{i}$.

Here, $x_{\perp} \in[-\epsilon, \epsilon]$ is the local coordinate in the direction perpendicular to $\Omega_{i}$ and $I_{\|}$is the $\left(n \times d_{i}\right)$ projection matrix onto the tangent bundle of $\Omega_{i}$. It follows that $\sigma_{i}$ is a $\left(n \times d_{i}\right)$ tensor field on $\Omega_{i}$.

Analogously, for $n=3$, the variable $\sigma_{i}$ is defined for $i \in I^{1}$ by integrating over both directions perpendicular to $\Omega_{i}$. Finally, for $i \in I^{0}$, there is no tangent bundle and it follows that $\sigma_{i}$ is undefined in the intersection points.

On the other hand, we define the displacement $\boldsymbol{u}_{i}$ in $\Omega_{i}$ by averaging the displacement $\tilde{\boldsymbol{u}}_{i}$ in $Y_{i}$ over the directions perpendicular to $\Omega_{i}$. In turn, $\boldsymbol{u}_{i}$ is naturally in $\mathbb{R}^{n}$ for all $i \in I$.

It becomes convenient to construct mixed-dimensional entities for these two variables by using a direct sum over the manifolds on which they are defined. We refer to these entities by omitting the subscript $i$, i.e.:

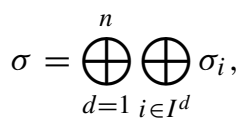

$$
\boldsymbol{u}=\bigoplus_{i \in I} \boldsymbol{u}_{i}
$$

\subsection{Mixed-dimensional equations}

With the given scaling from the previous subsection, let us consider the governing equations in the lowerdimensional manifolds. In this generalization to the mixeddimensional geometry, a structure similar to the system (3.2) is uncovered. We start by introducing the linear momentum balance equation, followed by the stress-strain relationships and finish with the conservation of angular momentum.

The balance of linear momentum (3.2b) is generalized first. After integrating the conservation law in the direction(s) normal to the inclusion (see e.g. [10, 19] for the analogue in fracture flow models), we obtain:

$\nabla_{i} \cdot \sigma_{i}-\left.\sum_{j \in \hat{J}_{i}}\left(\boldsymbol{n} \cdot \sigma_{\hat{\jmath}}\right)\right|_{\Gamma_{j}}=-\boldsymbol{f}_{i}, \quad$ in $\Omega_{i}, i \in \bigcup_{d=1}^{n-1} I^{d}$.

Here, $\boldsymbol{f}_{i}$ is the body force acting on $Y_{i}$, integrated over its cross-sectional measure. In other words, for $n=3$, we obtain $f_{i}$ by integrating over the cross-sectional length $\left(i \in I^{2}\right)$, area $\left(i \in I^{1}\right)$, or volume $\left(i \in I^{0}\right)$ of the physical inclusion $Y_{i}$.

The surficial divergence $\left(\nabla_{i} \cdot\right)$ on $\Omega_{i}$ and the normal trace operator $(\boldsymbol{n} \cdot)$ onto $\Gamma_{j}$ are applied row-wise. Hence, the divergence in $\Omega_{i}$ maps from $\mathbb{R}^{n \times d_{i}}$ to $\mathbb{R}^{n}$ and the normal trace on $\Gamma_{j}$ maps $\mathbb{R}^{n \times d_{\hat{\jmath}}}$ to $\mathbb{R}^{n}$.

For the zero-dimensional manifolds, there is no divergence operator or $\sigma_{i}$ available and the balance law is completely given by the sum of forces on $\Gamma^{1}$ :

$\left.\sum_{j \in \hat{J}_{i}}\left(\boldsymbol{n} \cdot \sigma_{\hat{\jmath}}\right)\right|_{\Gamma_{j}}=-\boldsymbol{f}_{i}, \quad$ in $\Omega_{i}, i \in I^{0}$.

To shorten notation, we introduce the jump operator $\llbracket \cdot \rrbracket_{i}$ which maps functions defined on the interface $\Gamma_{j}$ with $j \in$ $\hat{J}_{i}$ to the central manifold $\Omega_{i}$ such that

$\llbracket \phi \rrbracket_{i}=\left.\sum_{j \in \hat{J}_{i}} \phi\right|_{\Gamma_{j}}, \quad \forall i \in I$.

Following [10], we introduce the mixed-dimensional divergence operator $(\mathfrak{D} \cdot)$ and rewrite the conservation equation to the concise form:

$\mathfrak{D} \cdot \sigma:=\nabla \cdot \sigma-\llbracket \boldsymbol{n} \cdot \hat{\sigma} \rrbracket=-\boldsymbol{f}, \quad$ in $\Omega$.

We now continue by defining the stress-strain relationships in the lower-dimensional manifolds in analogy with Eq. 3.2a. For that, we first introduce the gradient operator $\mathfrak{D}$ as:

$\mathfrak{D} \boldsymbol{u}= \begin{cases}\nabla_{i} \boldsymbol{u}_{i}, & \text { in } \Omega_{i}, i \in \cup_{d=1}^{n} I^{d} \\ \check{\boldsymbol{u}}-\hat{\boldsymbol{u}}, & \text { on } \Gamma_{j}, \quad j \in J .\end{cases}$

We emphasize that the gradient $\nabla_{i}$ relates to the tangential direction(s) and is applied row-wise. Since we have $\mathfrak{D} \boldsymbol{u}$ 
defined on both $\Omega$ and $\Gamma$, we need to provide stress-strain relationships inside and on the boundaries of the domains.

The stress-strain relationships are then described by an operator $\mathfrak{A}$ acting on the stress on the manifolds $\Omega$ and the interfaces $\Gamma$ :

$\mathfrak{A} \sigma=\mathfrak{D} \boldsymbol{u}-\chi, \quad$ on $\Omega \times \Gamma$

with $\chi$ describing rotational motions, defined below. The operator $\mathfrak{A}$ is assumed to satisfy certain continuity and coercivity properties, presented in Eq. 4.2 in Section 4. The concise notation of Eq. 3.5 describes two relationships, one tangential to $\Omega$, and one normal to $\Gamma$. We therefore emphasize, using definition (3.4), that the above can be rewritten as:

$\mathfrak{A} \sigma:= \begin{cases}\mathfrak{A}_{\|} \sigma_{i}=\nabla_{i} \boldsymbol{u}_{i}-\chi_{i}, & \text { in } \Omega_{i}, i \in \cup_{d=1}^{n} I^{d} \\ \mathfrak{A}_{\perp} \sigma_{\hat{\jmath}}=\check{\boldsymbol{u}}-\hat{\boldsymbol{u}}, & \text { on } \Gamma_{j}, \quad j \in J .\end{cases}$

We let $\chi$ be the generalization of the variable $\chi_{i}$ from Section 3.1. Since in-plane rotations are only possible for $d \geq 2$, we let $\chi:=\left(\chi_{i}\right)_{i}$ with:

$$
\begin{array}{ll}
\chi_{i} \in \mathbb{R}_{\text {asym }}^{3 \times 3}, & i \in I^{3}, n=3, \\
\chi_{i} \in\left[\begin{array}{c}
\mathbb{R}_{\text {asym }}^{2 \times 2} \\
0
\end{array}\right] \subset \mathbb{R}^{3 \times 2}, & i \in I^{2}, n=3, \\
\chi_{i} \in \mathbb{R}_{\text {asym }}^{2 \times 2}, & i \in I^{2}, n=2 .
\end{array}
$$

We interpret $\chi_{i}=0$ for $i \in I^{0} \cup I^{1}$ and $\chi_{j}=0$ for $j \in J$.

Example 1 We provide an explicit example of $\mathfrak{A}$ using a fictitious material. In this material, we assume that the stress-strain relationships in tangential and normal directions are independent. This assumption leads to a model which captures in-plane shearing whereas out-ofplane stress components follow a one-dimensional Hooke's law. This behavior is described by the following constitutive laws:

$$
\begin{aligned}
& \sigma_{i}=V_{i}\left[\begin{array}{c}
2 \mu_{\|} \operatorname{sym}\left(\nabla_{i} \boldsymbol{u}_{\|, i}\right)+\lambda_{\|} \operatorname{Tr}\left(\nabla_{i} \boldsymbol{u}_{\|, i}\right) I \\
2 \mu_{\|} \nabla_{i} \boldsymbol{u}_{\perp, i}
\end{array}\right], \quad \text { in } \Omega_{i}, \quad \text { (3.6a) } \\
& \boldsymbol{n} \cdot \hat{\sigma}=\frac{1}{\epsilon_{j}}\left(2 \mu_{\perp}(\check{\boldsymbol{u}}-\hat{\boldsymbol{u}})+\lambda_{\perp}(\boldsymbol{n} \cdot(\check{\boldsymbol{u}}-\hat{\boldsymbol{u}})) \boldsymbol{n}\right), \quad \text { on } \Gamma_{j},(3.6 \mathrm{~b})
\end{aligned}
$$

Here, $\mu_{\|}$and $\lambda_{\|}$(respectively $\mu_{\perp}$ and $\lambda_{\perp}$ ) are the Lamé parameters describing the stress-strain relationship tangential (and normal) to the manifold. The parameter $V_{i}$ is the cross-sectional measure of the physical inclusion $Y_{i}$. On the other hand, $\epsilon_{j}$ is the distance from the central plane, line, or point of $Y_{i}$ to the interface with index $j$. For $j \in \hat{J}_{i}$ with $d_{i}=n-1$, it follows that $\epsilon_{j}$ is half the thickness of $Y_{i}$.
The inverse relations, mapping stresses to strains, are then given by:

$$
\begin{array}{rlrl}
\left.\left(\mathfrak{A}_{\|} \sigma\right)\right|_{\Omega_{i}} & =\frac{1}{2 \mu_{\|} V_{i}}\left(\sigma_{i}-\frac{\lambda_{\|}}{2 \mu_{\|}+d_{i} \lambda_{\|}} \operatorname{Tr}\left(\sigma_{i}\right) I_{i}\right), & & i \in \bigcup_{d=1}^{n} I^{d}, \\
\left.\left(\mathfrak{A}_{\perp} \sigma\right)\right|_{\Gamma_{j}} & =\frac{\epsilon_{i}}{2 \mu_{\perp}}\left(\boldsymbol{n} \cdot \sigma_{\hat{\jmath}}-\frac{\lambda_{\perp}}{2 \mu_{\perp}+\lambda_{\perp}}\left(\boldsymbol{n} \cdot \sigma_{\hat{\jmath}} \cdot \boldsymbol{n}\right) \boldsymbol{n}\right), & j \in J .
\end{array}
$$

Here, $I_{i} \in \mathbb{R}^{n \times d_{i}}$ is the tensor obtained by adding $\left(n-d_{i}\right)$ rows of zeroes to the $\left(d_{i} \times d_{i}\right)$ identity tensor.

Finally, we consider the symmetry of the stress tensor. Since the lower-dimensional manifolds model objects with finite width, the limit argument used to prove symmetry of the stress tensor is only valid within manifolds (and not transversely). Consequently, symmetry of the stress tensor is imposed within each manifold, expressed as:

$\operatorname{asym} \sigma=0$.

We remark that for $i \in I^{0} \cup I^{1}$, this equation is trivial since either $\sigma_{i}$ does not exist or is a vector. For $i \in$ $I^{2}$, this equation evaluates the asymmetry of the in-plane components.

Gathering Eqs. 3.3, 3.5, and 3.7, we arrive at the strong form of the generalized system of equations:

$$
\begin{aligned}
\mathfrak{A} \sigma-\mathfrak{D} \boldsymbol{u}+\chi & =0 & & \text { in } \Omega \times \Gamma, \\
\mathfrak{D} \cdot \sigma & =-\boldsymbol{f} & & \text { in } \Omega, \\
\operatorname{asym} \sigma & =0 & & \text { in } \Omega .
\end{aligned}
$$

To close the system, the boundary conditions are given by:

$\boldsymbol{n} \cdot \sigma=0$ on $\partial_{\sigma} \Omega, \quad \boldsymbol{u}=\boldsymbol{g}_{u}$ on $\partial_{u} \Omega$.

System (3.8) has a structure similar to Eq. 3.2 in that it is composed of constitutive law(s) complemented with a differential and an algebraic constraint. This structure is common in models concerning linear elasticity with relaxed symmetry $[2,5]$. We will show in the next section that the system indeed corresponds to a symmetric saddle-point problem.

\section{Variational formulation}

With the goal of obtaining a mixed finite element discretization, this section presents the weak formulation of the continuous problem. In order to do this, we introduce several analytical tools. First, the relevant function spaces are defined as well as the notational conventions concerning inner products. Next, we derive the variational formulation of Eq. 3.8 and show that it corresponds to a symmetric saddle point problem. 


\subsection{Function spaces}

The function spaces relevant for this problem are constructed as products of familiar function spaces on the $d$-dimensional manifolds. In particular, we define:

$\Sigma=\prod_{d=1}^{n} \prod_{i \in I^{d}}\left\{\tau_{i} \in\left(H\left(\operatorname{div}, \Omega_{i}\right)\right)^{n}: \begin{array}{l}\left.\boldsymbol{n} \cdot \tau_{i}\right|_{\partial_{\sigma} \Omega_{i}}=0, \\ \boldsymbol{n} \cdot \tau_{i} \mid \Gamma_{j} \in\left(L^{2}\left(\Gamma_{j}\right)\right)^{n}, \forall j \in \breve{J_{i}}\end{array}\right\}$

$\boldsymbol{U}=\prod_{d=0}^{n} \prod_{i \in I^{d}}\left(L^{2}\left(\Omega_{i}\right)\right)^{n}$,

$R=\prod_{d=2}^{n} \prod_{i \in I^{d}}\left(L^{2}\left(\Omega_{i}\right)\right)^{k_{d}}$,

where $\Sigma$ denotes the function space for the stress, $\boldsymbol{U}$ contains the displacement, and $R$ is the function space for the Lagrange multiplier enforcing symmetry of the stress tensor. The exponent $k_{d}$ is given by $k_{d}=\left(\begin{array}{l}d \\ 2\end{array}\right)=d(d-1) / 2$; see e.g. $[2,5]$.

The mixed-dimensional $L^{2}$-inner products on $\Omega$ and $\Gamma$ are defined as the sum of inner products over all corresponding manifolds:

$$
(f, g)_{\Omega}=\sum_{i \in I}\left(f_{i}, g_{i}\right)_{\Omega_{i}}, \quad(\phi, \varphi)_{\Gamma}=\sum_{i \in I} \sum_{j \in \hat{J}_{i}}\left(\phi_{i}, \varphi_{i}\right)_{\Gamma_{i}}
$$

Here, the implicit assumption is made that the contribution is zero for all manifolds on which $f$ is undefined. For example, for $\sigma, \tau \in \Sigma$, the inner product $(\sigma, \tau)_{\Omega}$ has no contribution on $\Omega^{0}$. Likewise for functions in $R$, the inner product is zero on manifolds $\Omega_{i}$ with $i \in I^{0} \cup I^{1}$.

For functions $\sigma, \tau \in \Sigma$, we note that they are defined on both $\Omega$ and $\Gamma$. For convenience, we introduce the combined inner product:

$(\sigma, \tau)_{\Omega \times \Gamma}:=(\sigma, \tau)_{\Omega}+(\boldsymbol{n} \cdot \sigma, \boldsymbol{n} \cdot \tau)_{\Gamma}$,

which, in the case of the operator $\mathfrak{A}$, is understood as:

$(\mathfrak{A} \sigma, \tau)_{\Omega \times \Gamma}:=\left(\mathfrak{A}_{\|} \sigma, \tau\right)_{\Omega}+\left(\mathfrak{A}_{\perp} \sigma, \boldsymbol{n} \cdot \tau\right)_{\Gamma}$.

The inner products naturally induce the $L^{2}$-type norms $\|\cdot\|_{\Omega},\|\cdot\|_{\Gamma}$, and $\|\cdot\|_{\Omega \times \Gamma}$. With these norms, we assume that $\mathfrak{A}$ is continuous and coercive with respect to the norm $\|\cdot\|_{\Omega \times \Gamma}$. Thus, for all $\sigma, \tau \in \Sigma$, we have:

$(\mathfrak{A} \sigma, \tau)_{\Omega \times \Gamma} \lesssim\|\sigma\|_{\Omega \times \Gamma}\|\tau\|_{\Omega \times \Gamma} \quad(\mathfrak{A} \sigma, \sigma)_{\Omega \times \Gamma} \gtrsim\|\sigma\|_{\Omega \times \Gamma}^{2}$

The relation $a \lesssim b$ (respectively $\gtrsim$ ) implies that a constant $C>0$ exists, independent of the mesh size $h$, such that $a \leq C b$ (respectively $\geq$ ).

\subsection{Identifying the symmetric saddle point problem}

In this section, we make two key observations which allow us to derive a variational formulation of Eq. 3.8 which is symmetric. First, let us consider the terms containing $\boldsymbol{u}$ in the stress-strain relationships (3.8a) and (3.8a). We multiply these terms with $\tau \in \Sigma$ and $\boldsymbol{n} \cdot \tau \in L^{2}(\Gamma)$, respectively, and integrate to obtain the following integration by parts formula:

$$
\begin{aligned}
(\mathfrak{D} \boldsymbol{u}, \tau)_{\Omega \times \Gamma}= & (\nabla \boldsymbol{u}, \tau)_{\Omega}+((\check{\boldsymbol{u}}-\hat{\boldsymbol{u}}), \boldsymbol{n} \cdot \hat{\tau})_{\Gamma} \\
= & -(\boldsymbol{u}, \nabla \cdot \tau)_{\Omega}+(\boldsymbol{u}, \boldsymbol{n} \cdot \tau)_{\partial_{u} \Omega}+(\hat{\boldsymbol{u}}, \boldsymbol{n} \cdot \hat{\tau})_{\Gamma} \\
& +(\check{\boldsymbol{u}}, \boldsymbol{n} \cdot \hat{\tau})_{\Gamma}-(\hat{\boldsymbol{u}}, \boldsymbol{n} \cdot \hat{\tau})_{\Gamma} \\
= & -(\boldsymbol{u}, \nabla \cdot \tau)_{\Omega}+(\boldsymbol{u}, \llbracket \boldsymbol{n} \cdot \hat{\tau} \rrbracket)_{\Omega}+(\boldsymbol{u}, \boldsymbol{n} \cdot \tau)_{\partial_{u} \Omega} \\
= & -(\boldsymbol{u}, \mathfrak{D} \cdot \tau)_{\Omega}+(\boldsymbol{u}, \boldsymbol{n} \cdot \tau)_{\partial_{u} \Omega} .
\end{aligned}
$$

Here, $\mathfrak{D}$. is the mixed-dimensional divergence operator from Eq. 3.3.

Secondly, we introduce the operator skw which evaluates the asymmetric part of a matrix. More specifically, for a matrix $B \in \mathbb{R}^{n \times d}$ with components $b_{i j}$, let

$\operatorname{skw} B:= \begin{cases}s k w_{3} B=\left[b_{23}-b_{32}, b_{31}-b_{13}, b_{12}-b_{21}\right]^{T}, & d=3, \\ s k w_{2} B=b_{12}-b_{21}, & d=2 .\end{cases}$

This operator is naturally lifted to skw : $\Sigma \rightarrow R$. Next, we turn our attention to the term in Eq. 3.8a containing the asymmetric variable $\chi$. Let us multiply this term with $\tau \in \Sigma$ and integrate over $\Omega^{2} \cup \Omega^{3}$. With the introduction of $r=\frac{1}{2} \mathrm{skw} \chi \in R$, we obtain:

$(\chi, \tau)_{\Omega}=(r, \operatorname{skw} \tau)_{\Omega}$.

By employing test functions $(\tau, \boldsymbol{v}, s) \in \Sigma \times \boldsymbol{U} \times R$, the integration by parts formula (4.3), and the operator skw, we obtain the following variational formulation of the problem (3.8):

Find $(\sigma, \boldsymbol{u}, r) \in \Sigma \times \boldsymbol{U} \times R$ such that

$$
\begin{aligned}
(\mathfrak{A} \sigma, \tau)_{\Omega \times \Gamma}+(\boldsymbol{u}, \mathfrak{D} \cdot \tau)_{\Omega}+(r, \operatorname{skw} \tau)_{\Omega} & =\left(\boldsymbol{g}_{\boldsymbol{u}}, \boldsymbol{n} \cdot \tau\right)_{\partial_{u} \Omega}, & & \tau \in \Sigma,(4.4 \mathrm{a}) \\
(\mathfrak{D} \cdot \sigma, \boldsymbol{v})_{\Omega} & =-(\boldsymbol{f}, \boldsymbol{v})_{\Omega}, & & \boldsymbol{v} \in \boldsymbol{U},(4.4 \mathrm{~b}) \\
(\operatorname{skw} \sigma, s)_{\Omega} & =0, & & s \in R .(4.4 \mathrm{c})
\end{aligned}
$$

We identify system (4.4) as a saddle point problem by introducing the bilinear forms $a: \Sigma \times \Sigma \rightarrow \mathbb{R}$ and $b: \Sigma \times(\boldsymbol{U} \times R) \rightarrow \mathbb{R}:$

$$
\begin{aligned}
a(\sigma ; \tau) & :=(\mathfrak{A} \sigma, \tau)_{\Omega \times \Gamma} \\
b(\sigma ; \boldsymbol{v}, s) & :=(\mathfrak{D} \cdot \sigma, \boldsymbol{v})_{\Omega}+(\operatorname{skw} \sigma, s)_{\Omega} .
\end{aligned}
$$

The problem (4.4) can then be rewritten to the following, equivalent formulation: 
Find $(\sigma, \boldsymbol{u}, r) \in \Sigma \times \boldsymbol{U} \times R$ such that

$$
\begin{aligned}
a(\sigma ; \tau)+b(\tau ; \boldsymbol{u}, r) & =\left(\boldsymbol{g}_{u}, \boldsymbol{n} \cdot \tau\right)_{\partial_{u} \Omega} \\
b(\sigma ; \boldsymbol{v}, s) & =-(\boldsymbol{f}, \boldsymbol{v})_{\Omega}
\end{aligned}
$$

for all $(\tau, \boldsymbol{v}, s) \in \Sigma \times \boldsymbol{U} \times R$.

\section{Well-posedness}

In this section, we show well-posedness of the continuous formulation (4.4). The key is to associate appropriate norms to the composite function spaces introduced in the previous section. In the mixed-dimensional setting considered here, let us endow $\Sigma, \boldsymbol{U}$, and $R$ with the following norms:

$$
\begin{aligned}
\|\tau\|_{\Sigma} & =\left(\|\tau\|_{\Omega}^{2}+\|\boldsymbol{n} \cdot \tau\|_{\Gamma}^{2}+\|\mathfrak{D} \cdot \tau\|_{\Omega}^{2}\right)^{1 / 2}, \\
\|\boldsymbol{v}\|_{U} & =\|\boldsymbol{v}\|_{\Omega}, \\
\|s\|_{R} & =\|s\|_{\Omega} .
\end{aligned}
$$

The proof of well-posedness consists of proving sufficient conditions on the bilinear forms $a$ and $b$ from Eq. 4.5 to invoke standard saddle-point theory. First, we show continuity of the operators, followed by ellipticity of $a$ and inf-sup on $b$.

Lemma 1 (Continuity) The bilinear forms $a$ and $b$ from Eq. 4.5 are continuous with respect to the norms given by Eq. 5.1.

Proof The continuity of $a$ follows from Eq. 4.2. For the blinear form $b$, we derive:

$$
\begin{aligned}
b(\sigma ; \boldsymbol{v}, s) & =(\mathfrak{D} \cdot \sigma, \boldsymbol{v})_{\Omega}+(\operatorname{skw} \sigma, s)_{\Omega} \\
& \leq\|\mathfrak{D} \cdot \sigma\|_{\Omega}\|\boldsymbol{v}\|_{\Omega}+\|\sigma\|_{\Omega}\|s\|_{\Omega} \\
& \lesssim\|\sigma\|_{\Sigma}\left(\|\boldsymbol{v}\|_{U}+\|s\|_{R}\right) .
\end{aligned}
$$

Next, we focus on the bilinear form $a$. For the purposes of our analysis, it suffices to show that $a$ is elliptic on a specific subspace of $\Sigma$ generated by $b$. This is formally considered in the following lemma.

Theorem 1 (Ellipticity) Given the bilinear forms $a$ and $b$ from Eq. 4.5. If $\sigma \in \Sigma$ satisfies

$b(\sigma ; \boldsymbol{v}, s)=0, \quad$ for all $(\boldsymbol{v}, s) \in \boldsymbol{U} \times R$,

then the following ellipticity bound holds

$a(\sigma ; \sigma) \gtrsim\|\sigma\|_{\Sigma}^{2}$.
Proof We set $s=0$ in condition (5.2). The assumption holds for all $\boldsymbol{v} \in \boldsymbol{U}$, thus noting that $\mathfrak{D} \cdot \sigma \in \prod_{i \in I} L^{2}\left(\Omega_{i}\right)=$ $\boldsymbol{U}$, we obtain:

$\|\mathfrak{D} \cdot \sigma\|_{\Omega}=0$,

The proof is concluded by combining (5.3) with the coercivity of $\mathfrak{A}$ from Eq. 4.2.

With the properties of $a$ proven, we continue by considering an inf-sup condition on the bilinear form $b$. This is shown in the following theorem, which relies on the constructions from Lemmas 2 and 4, presented afterwards.

Theorem 2 (Inf-Sup) The bilinear form $b$ satisfies for all $(\boldsymbol{u}, r) \in \boldsymbol{U} \times R$,

$\sup _{\tau \in \Sigma} \frac{b(\tau ; \boldsymbol{u}, r)}{\|\tau\|_{\Sigma}} \gtrsim\|\boldsymbol{u}\|_{U}+\|r\|_{R}$.

Proof The proof consists of constructing a suitable $\tau \in \Sigma$ for a given pair $(\boldsymbol{u}, r) \in \boldsymbol{U} \times R$. Its construction is based on constructing two auxiliary functions $\eta, \xi \in \Sigma$ using the techniques from Lemmas 2 and 4. Setting $\tau$ as the sum of these two functions then yields the result.

First, Lemma 2 allows us to construct $\eta \in \Sigma$ such that

$\mathfrak{D} \cdot \eta=\boldsymbol{u}, \quad\|\eta\|_{\Sigma} \lesssim\|\boldsymbol{u}\|_{U}$.

Secondly, we choose $\xi \in \Sigma$ using Lemma 4 with given $(r-\operatorname{skw} \eta) \in R$ such that

$$
\begin{aligned}
\mathrm{skw} \xi & =r-\mathrm{skw} \eta \\
\mathfrak{D} \cdot \xi & =0 \\
\|\xi\|_{\Sigma} & \lesssim\|r\|_{R}+\|\mathrm{skw} \eta\|_{R} \leq\|r\|_{R}+\|\eta\|_{\Sigma} .
\end{aligned}
$$

By setting $\tau=\eta+\xi$, it follows that:

$$
\begin{aligned}
b(\tau ; \boldsymbol{u}, r) & =(\mathfrak{D} \cdot \tau, \boldsymbol{u})_{\Omega}+(\operatorname{skw} \tau, r)_{\Omega} \\
& =(\mathfrak{D} \cdot \eta, \boldsymbol{u})_{\Omega}+(\operatorname{skw} \eta+\operatorname{skw} \xi, r)_{\Omega} \\
& =\|\boldsymbol{u}\|_{U}^{2}+\|r\|_{R}^{2}
\end{aligned}
$$

Furthermore, the bound on $\tau$ is derived using Eqs. 5.4 and $5.5 \mathrm{c}$ :

$\|\tau\|_{\Sigma} \leq\|\eta\|_{\Sigma}+\|\xi\|_{\Sigma} \lesssim\|\boldsymbol{u}\|_{U}+\|r\|_{R}$.

The proof is concluded by combining Eqs. 5.6 and 5.7.

Lemma 2 For each $\boldsymbol{u} \in \boldsymbol{U}$, a function $\eta \in \Sigma$ exists such that

$\mathfrak{D} \cdot \eta=\boldsymbol{u}, \quad\|\eta\|_{\Sigma} \lesssim\|\boldsymbol{u}\|_{U}$

Proof Considering $\boldsymbol{u} \in \boldsymbol{U}$ given, the function $\eta$ is constructed hierarchically. For each dimension $d$, we first 
set an interface value $\phi$ on $\Gamma^{d}$, followed by a suitable extension into $\Omega^{d}$.

0 . Given $i \in I^{0}$, we construct the adjacent interface functions $\boldsymbol{\phi}_{j} \in L^{2}\left(\Gamma_{j}\right)$ such $\boldsymbol{\phi}_{j}=-\boldsymbol{u}_{i}$ for a chosen $j \in \hat{J}_{i}$ and zero for all other $j \in \hat{J}_{i}$. Repeating this construction for all $i \in I^{0}$, it follows that:

$$
\begin{aligned}
-\llbracket \boldsymbol{\phi} \rrbracket_{i} & =\boldsymbol{u}_{i}, \quad \forall i \in I^{0} \\
\|\boldsymbol{\phi}\|_{\Gamma^{0}} & =\|\boldsymbol{u}\|_{\Omega^{0}} .
\end{aligned}
$$

1. We continue with $i \in I^{1}$ and perform the following two steps. First, the function $\eta_{i}$ is constructed as the bounded $H\left(\operatorname{div}, \Omega_{i}\right)$-extension of the given $\phi_{j}$ with $j \in \check{J}_{i}$. We use the extension operator as described in [23] (Section 4.1.2), giving us the properties:

$$
\begin{aligned}
\left.\left(\boldsymbol{n} \cdot \eta_{i}\right)\right|_{\Gamma_{j}} & =\boldsymbol{\phi}_{j}, \quad \forall j \in \check{J}_{i}, \\
\left.\left(\boldsymbol{n} \cdot \eta_{i}\right)\right|_{\partial \Omega_{i} \backslash \Gamma^{0}} & =0, \\
\left\|\eta_{i}\right\|_{\Omega_{i}}+\left\|\nabla \cdot \eta_{i}\right\|_{\Omega_{i}} & \lesssim \sum_{j \in \breve{J}_{i}}\|\boldsymbol{\phi}\|_{\Gamma_{j}} .
\end{aligned}
$$

Secondly, we further define $\phi$ on $\Gamma_{j}$ with $j \in \hat{J}_{i}$. We choose a single $j \in \hat{J}_{i}$ and set $\boldsymbol{\phi}_{j}=-\boldsymbol{u}_{i}+\nabla \cdot \eta_{i}$. For all other $j \in \hat{J}_{i}$, we set $\boldsymbol{\phi}_{j}=0$. It then immediately follows that:

$-\llbracket \boldsymbol{\phi} \rrbracket_{i}=\boldsymbol{u}_{i}-\nabla \cdot \eta_{i}$,

Repeating these two steps for all $i \in I^{1}$ gives us the bound:

$$
\begin{aligned}
\|\boldsymbol{\phi}\|_{\Gamma^{1}} & \leq\|\boldsymbol{u}\|_{\Omega^{1}}+\|\nabla \cdot \eta\|_{\Omega^{1}} \\
& \lesssim\|\boldsymbol{u}\|_{\Omega^{1}}+\|\boldsymbol{\phi}\|_{\Gamma^{0}}
\end{aligned}
$$

in which the final inequality follows from Eq. $5.10 \mathrm{c}$.

2. For $n=3$, repeat the previous step for all $i \in I^{2}$ to obtain $\eta_{i}$ and $\boldsymbol{\phi}_{j}$ for all $j \in \hat{J}_{i}$.

3. The construction of $\eta$ is finalized with its topdimensional components $\eta_{i}$ with $i \in I^{n}$. Let the pair $\left(\eta_{i}, \tilde{\boldsymbol{v}}_{i}\right) \in\left(H\left(\operatorname{div}, \Omega_{i}\right)\right)^{n} \times\left(L^{2}\left(\Omega_{i}\right)\right)^{n}$ be the weak solution to the Poisson problem:

$$
\begin{aligned}
\eta_{i}+\nabla \tilde{\boldsymbol{v}}_{i} & =0, \\
\nabla \cdot \eta_{i} & =\boldsymbol{u}_{i}, \\
\left.\left(\boldsymbol{n} \cdot \eta_{i}\right)\right|_{\Gamma_{j}} & =\boldsymbol{\phi}_{j}, \quad \quad j \in \check{\boldsymbol{J}}_{i}, \\
\left.\left(\boldsymbol{n} \cdot \eta_{i}\right)\right|_{\partial_{\sigma} \Omega_{i}} & =0, \\
\left.\left(\tilde{\boldsymbol{v}}_{i}\right)\right|_{\partial_{u} \Omega_{i}} & =0 .
\end{aligned}
$$

This problem is solved for all $i \in I^{n}$. We then exploit the elliptic regularity of Eq. 5.13 (see e.g. [14]) to obtain$$
\|\eta\|_{\Omega^{n}}+\|\nabla \cdot \eta\|_{\Omega^{n}} \lesssim\|\boldsymbol{\phi}\|_{\Gamma^{n-1}}+\|\boldsymbol{u}\|_{\Omega^{n}} .
$$

With $\eta$ constructed, we consider its two main properties. First, by Eqs. 5.9a, 5.11, and 5.13b, we have:

$\mathfrak{D} \cdot \eta=\boldsymbol{u}$.
Secondly, we find the following bound from Eqs. 5.9b, 5.10c, 5.12, 5.14, and 5.15a:

$$
\begin{aligned}
\|\eta\|_{\Sigma}^{2} & =\|\eta\|_{\Omega}^{2}+\|\boldsymbol{n} \cdot \eta\|_{\Gamma}^{2}+\|\mathfrak{D} \cdot \eta\|_{\Omega}^{2} \\
& \lesssim\|\boldsymbol{\phi}\|_{\Gamma}^{2}+\|\boldsymbol{u}\|_{\Omega}^{2} \\
& =\|\boldsymbol{u}\|_{U}^{2},
\end{aligned}
$$

thereby concluding the proof.

Before introducing the second ingredient used in the proof of Theorem 2, we require several key analytical tools, organized in the following diagram:

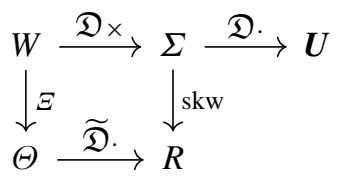

The function spaces $(\Theta$ and $W)$ and mappings $(\Xi, \widetilde{\mathfrak{D}}$, and $\mathfrak{D} \times)$ are defined next. Let the auxiliary space $\Theta$ be given by:

$\Theta=\prod_{d=2}^{3} \prod_{i \in I^{d}}\left(H^{1}\left(\Omega_{i}\right)\right)^{k_{d} \times d}$.

We emphasize that for an element $\theta \in \Theta$, this definition implies that $\theta_{i}$ is a 2 -vector for $i \in I^{2}$ and a $3 \times 3$ tensor for $i \in I^{3}$. Next, we follow [5] by introducing the mapping $\Xi$ and its right-inverse $\Xi^{-1}$ as:

$$
\begin{aligned}
\left.(\Xi w)\right|_{\Omega_{i}} & = \begin{cases}\left(w_{i}\right)^{T}-\operatorname{Tr}\left(w_{i}\right) I, & i \in I^{3}, \\
\left(w_{i, \|}\right)^{T}, & i \in I^{2},\end{cases} \\
\left.\left(\Xi^{-1} \theta\right)\right|_{\Omega_{i}} & = \begin{cases}\left(\theta_{i}\right)^{T}-\frac{1}{2} \operatorname{Tr}\left(\theta_{i}\right) I, & i \in I^{3}, \\
{\left[\theta_{i}, 0\right]^{T},} & i \in I^{2},\end{cases}
\end{aligned}
$$

with $w_{i, \|}$ the tangential components of $w_{i}$ with respect to $\Omega_{i} . W$ is defined as the space of functions that lie in the image of the inverse operator and have a mixed-dimensional curl in $\Sigma$, i.e.:

$W:=\left\{w \in \Xi^{-1} \Theta: \mathfrak{D} \times w \in \Sigma\right\}$.

We remark that for $w \in W, w_{i}$ is a 3 -vector for $i \in I^{3}$ and a $3 \times 3$ tensor for $i \in I^{3}$.

Next, we introduce a divergence-like operator $\widetilde{\mathfrak{D}} \cdot: \rightarrow \rightarrow$ $R$ given by:

$\tilde{\mathfrak{D}} \cdot \theta= \begin{cases}\nabla \cdot \theta_{i}, & i \in I^{3}, \\ \nabla \cdot \theta_{i}-\check{\boldsymbol{n}}_{i} \cdot \llbracket \boldsymbol{n} \cdot \hat{\theta} \rrbracket_{i}, & i \in I^{2} .\end{cases}$

Here, $\check{\boldsymbol{n}}_{i}$ is the unique unit vector normal to $\Omega_{i}$ that forms a positive orientation with the chosen basis of the tangential bundle. By definition, this divergence operator maps from $\Theta$ to $R$, and we emphasize that $\widetilde{\mathfrak{D}} \cdot \theta$ is a vector for $d=3$ and a scalar for $d=2$. 
Finally, the mixed-dimensional curl $(\mathfrak{D} \times)$ of $w \in W$ (see e.g. $[9,18])$ is given by:

$\mathfrak{D} \times w=\left\{\begin{aligned} \nabla \times w_{i}, & \forall i \in I^{3}, \\ \nabla^{\perp} w_{i}-\llbracket \boldsymbol{n} \times \hat{w} \rrbracket_{i}, & \forall i \in I^{2}, \\ -\llbracket \boldsymbol{n}^{\perp} \hat{w} \rrbracket_{i}, & \forall i \in I^{1} .\end{aligned}\right.$

Here, the superscript $\perp$ implies $\left[v_{1}, v_{2}\right]^{\perp}=\left[-v_{2}, v_{1}\right]$, e.g., $\nabla^{\perp}$ is a rotated gradient operator. We note that all differential operations are performed row-wise. Hence, for $n=3$, the mixed-dimensional curl maps to a $3 \times 3$ tensor in $\Omega^{3}$, a $3 \times 2$ tensor in $\Omega^{2}$ (in local coordinates) and a 3 -vector in $\Omega^{1}$ (in local coordinates). Thus, an exact correspondence with the function space $\Sigma$ is obtained, as reflected in the diagram.

Lemma 3 The operators in diagram (5.16) enjoy the following two properties for all $w \in W$ :

$$
\mathfrak{D} \cdot \mathfrak{D} \times w=0, \quad \quad \operatorname{skw\mathfrak {D}} \times w=\tilde{\mathfrak{D}} \cdot \Xi w .
$$

Proof The top row of Eq. 5.16 uses the differential operators from the mixed-dimensional De Rham complex [9]. The first equality then follows from the fact that exact forms are closed. It remains to show commutativity. By the definition of $\Xi$ (see e.g. $[5,8]$ ), we have:

$$
\begin{array}{rlrl}
\operatorname{skw}_{3} \nabla \times w_{i} & =\nabla \cdot \Xi_{3} w_{i}, & \forall i & \in I^{3}, \\
\operatorname{skw}_{2} \nabla^{\perp} w_{i} & =\nabla \cdot \Xi_{2} w_{i}, & \forall i \in I^{2},
\end{array}
$$

in which the subscript $d$ denotes a restriction of the operator to $\Omega^{d}$. Furthermore, we note that on $\Omega_{i}$ with $i \in I^{2}$, the skw operator evaluates the asymmetry with respect to the tangent bundle of $\Omega_{i}$. In turn, we have for $M \in \mathbb{R}^{3 \times 3}$ that $\left.\left(\operatorname{skw}_{2} M\right)\right|_{\Omega_{i}}=\check{\boldsymbol{n}}_{i} \cdot \mathrm{skw}_{3} M$. This gives us:

$$
\begin{aligned}
& \left.(\operatorname{skw} \mathfrak{D} \times w)\right|_{\Omega_{i}}=\operatorname{skw}_{3} \nabla \times w_{i} \\
& =\nabla \cdot \Xi_{3} w_{i}=\left.(\tilde{\mathfrak{D}} \cdot \Xi w)\right|_{\Omega_{i}}, \\
& \left.(\operatorname{skw} \mathfrak{D} \times w)\right|_{\Omega_{i}}=\operatorname{skw}_{2}\left(\nabla^{\perp} w_{i}-\llbracket \boldsymbol{n} \times \hat{w} \rrbracket_{i}\right) \\
& =\nabla \cdot \Xi_{2} w_{i}-\check{\boldsymbol{n}}_{i} \cdot \llbracket \boldsymbol{n} \cdot \Xi_{3} \hat{w} \rrbracket_{i} \\
& =\left.(\widetilde{\mathfrak{D}} \cdot \Xi w)\right|_{\Omega_{i}},
\end{aligned}
$$

$\forall i \in I^{3}$,

$\forall i \in I^{2}$.

Lemma 4 Given $r \in R$, a function $\xi \in \Sigma$ exists such that

$$
\mathfrak{D} \cdot \xi=0, \quad \operatorname{skw} \xi=r, \quad\|\xi\|_{\Sigma} \lesssim\|r\|_{R} .
$$

Proof We give the proof for $n=3$, the case $n=2$ being simpler. The strategy is to exploit the properties shown in Lemma 3 and first construct a bounded $\theta \in \Theta$ such that $\widetilde{\mathfrak{D}} \cdot \theta=r$. Then, by setting $w=\Xi^{-1} \theta$ and $\xi=\mathfrak{D} \times w$, we obtain two of the desired properties:

$$
\mathfrak{D} \cdot \xi=\mathfrak{D} \cdot \mathfrak{D} \times w=0, \quad \operatorname{skw} \xi=\operatorname{skw} \mathfrak{D} \times w=\tilde{\mathfrak{D}} \cdot \theta=r .
$$

The estimate will then follow from the boundedness of $\theta$.

The construction of $\theta$ proceeds according to the following three steps: we first construct an interface function $\phi \in H^{1}\left(\Gamma^{2}\right)$ which then serves as a source function for $\theta_{i}$ with $i \in I^{2}$ and as a boundary condition for $\theta_{i}$ with $i \in I^{3}$.

1. We start by defining a scalar function $\phi$ in the trace space $H^{1}\left(\Gamma^{2}\right)$. We let $\phi$ vanish at all intersections and extremities, i.e. $\phi$ is in the function space $\Phi$ given by:

$$
\Phi:=\prod_{i \in I^{2}} \prod_{j \in \hat{J}_{i}} H_{0}^{1}\left(\Gamma_{j}\right)
$$

Now, let $\phi$ be the solution to the following minimization problem:

$\min _{\varphi \in \Phi} \frac{1}{2}\|\varphi\|_{H^{1}\left(\Gamma^{2}\right)}^{2} \quad$ subject to $\Pi_{\mathbb{R}_{i}}\left(\llbracket \varphi \rrbracket_{i}+r_{i}\right)=0, \forall i \in I^{2}$.

with $\Pi_{\mathbb{R}_{i}}$ the projection onto constants on $\Omega_{i}$. Due to the regularity of this problem and the imposed constraint, we have:

$$
\begin{aligned}
-\Pi_{\mathbb{R}_{i}} \llbracket \phi \rrbracket_{i} & =\Pi_{\mathbb{R}_{i}} r_{i}, \quad \forall i \in I^{2} \\
\|\phi\|_{H^{1}\left(\Gamma^{2}\right)} & \lesssim\|r\|_{\Omega^{2}} .
\end{aligned}
$$

2. For each $i \in I^{2}$, we construct a function $\theta_{i}$ using $\phi$ as a source function. Specifically, let $\left(\theta_{i}, p_{i}\right) \in$ $\left(H_{0}^{1}\left(\Omega_{i}\right)\right)^{2} \times L^{2}\left(\Omega_{i}\right)$ be the weak solution to the Stokes problem:

$$
\begin{aligned}
\nabla \cdot\left(\nabla \theta_{i}\right)-\nabla p_{i} & =0 \\
\nabla \cdot \theta_{i} & =\left(I-\Pi_{\mathbb{R}_{i}}\right)\left(r_{i}+\llbracket \phi \rrbracket_{i}\right), \\
\left.\left(\theta_{i}\right)\right|_{\partial \Omega_{i}} & =0 .
\end{aligned}
$$

The following bound is then satisfied from the regularity of Eq. 5.27a (see e.g. [14]) combined with Eq. 5.26b:

$$
\|\theta\|_{H^{1}\left(\Omega^{2}\right)} \lesssim\|r\|_{\Omega^{2}}+\|\phi\|_{\Gamma^{2}} \lesssim\|r\|_{\Omega^{2}}
$$

3. To finalize $\theta \in \Theta$, we create $\theta_{i}$ for $i \in I^{3}$ using $\phi$ from the first step as a boundary condition. Let $\theta_{i} \in$ $\left(H^{1}\left(\Omega_{i}\right)\right)^{3 \times 3}$ and an auxiliary pressure variable $p_{i} \in$ $\left(L^{2}\left(\Omega_{i}\right)\right)^{3}$ be the weak solution to the following Stokes problem:

$$
\begin{aligned}
\nabla \cdot\left(\nabla \theta_{i}\right)-\nabla p_{i} & =0, \\
\nabla \cdot \theta_{i} & =r_{i}, \\
\left.\left(\theta_{i}\right)\right|_{\Gamma_{j}} & =\phi_{j} \check{\boldsymbol{n}}_{i} \boldsymbol{n}_{j}^{T}, \quad j \in \check{J}_{i}, \\
\left.\left(p_{i}\right)\right|_{\partial \Omega_{i} \backslash \Gamma^{2}} & =0 .
\end{aligned}
$$

Recall that $\check{\boldsymbol{n}}_{i}$, the unique normal vector of $\Omega_{i}$, and $\boldsymbol{n}_{j}$, the normal vector defined on $\Gamma_{j}$, are equal up to sign. This problem is well-posed since $\partial \Omega_{i} \backslash \Gamma^{2}$ has positive measure, for each $i \in I^{3}$, by assumption. We 
have the following bound due to the regularity of the Stokes problems:

$$
\|\theta\|_{H^{1}\left(\Omega^{3}\right)} \lesssim\|r\|_{\Omega^{3}}+\|\phi\|_{\Gamma^{2}} \lesssim\|r\|_{\Omega} .
$$

Combining all $\theta_{i}$ from the final two steps gives us $\theta \in \Theta$. We first note that the properties Eqs. 5.26a, 5.27b, and 5.29b result in:

$$
\begin{aligned}
\left.(\widetilde{\mathfrak{D}} \cdot \theta)\right|_{\Omega_{i}} & =\nabla \cdot \theta_{i}-\check{\boldsymbol{n}}_{i} \cdot \llbracket \boldsymbol{n} \cdot \theta_{i} \rrbracket & & \\
& =\left(I-\Pi_{\mathbb{R}_{i}}\right)\left(r_{i}+\llbracket \phi \rrbracket_{i}\right)-\llbracket \phi \rrbracket_{i}=r_{i}, & & \forall i \in I^{2}, \\
\left.(\widetilde{\mathfrak{D}} \cdot \theta)\right|_{\Omega_{i}} & =\nabla \cdot \theta_{i}=r_{i}, & & \forall i \in I^{3} .
\end{aligned}
$$

Hence, we have $\widetilde{\mathfrak{D}} \cdot \theta=r$ and it follows from Eq. 5.23 that setting $w=\Xi^{-1} \theta$ and $\xi=\mathfrak{D} \times w$ provides the first two properties. The bound follows due to Eqs. 5.26b, 5.28, and 5.30:

$$
\begin{aligned}
\|\xi\|_{\Sigma}^{2} & =\|\mathfrak{D} \times w\|_{\Omega}^{2}+\|\boldsymbol{n} \cdot(\mathfrak{D} \times w)\|_{\Gamma}^{2} \\
& \lesssim\|w\|_{H^{1}(\Omega)}^{2}+\|\hat{w}\|_{H^{1}(\Gamma)}^{2} \\
& \lesssim\|\theta\|_{H^{1}(\Omega)}^{2}+\|\hat{\theta}\|_{H^{1}\left(\Gamma^{2}\right)}^{2} \\
& =\|\theta\|_{H^{1}(\Omega)}^{2}+\|\phi\|_{H^{1}\left(\Gamma^{2}\right)}^{2} \\
& \lesssim\|r\|_{R}^{2},
\end{aligned}
$$

as desired.

With the proven properties of the bilinear forms $a$ and $b$, the main result of this section is summarized by the following theorem:

Theorem 3 Problem (4.4) is well-posed with respect to the norms (5.1). That is, a unique solution exists satisfying the bound:

$\|\sigma\|_{\Sigma}+\|\boldsymbol{u}\|_{U}+\|r\|_{R} \lesssim\left\|\boldsymbol{g}_{u}\right\|_{H^{\frac{1}{2}\left(\partial_{u} \Omega\right)}}+\|\boldsymbol{f}\|_{\Omega}$

Proof It suffices to show continuity of the right-hand side of Eq. 4.4 with respect to the norms above. For that purpose, we derive the following bound using Cauchy-Schwarz and a trace inequality:

$$
\begin{aligned}
\left(\boldsymbol{g}_{u}, \boldsymbol{n} \cdot \tau\right)_{\partial_{u} \Omega}-(\boldsymbol{f}, \boldsymbol{v})_{\Omega} \lesssim & \left\|\boldsymbol{g}_{u}\right\|_{H^{\frac{1}{2}}\left(\partial_{u} \Omega\right)}\|\tau\|_{H(\operatorname{div}, \Omega)}+\|\boldsymbol{f}\|_{\Omega}\|\boldsymbol{v}\|_{U} \\
\lesssim & \left\|\boldsymbol{g}_{u}\right\|_{H^{\frac{1}{2}}\left(\partial_{u} \Omega\right)}\left(\|\tau\|_{\Omega}+\|\mathfrak{D} \cdot \tau\|_{\Omega}\right. \\
& \left.+\|\llbracket \boldsymbol{n} \cdot \tau \rrbracket\|_{\Omega}\right)+\|\boldsymbol{f}\| \Omega\|\boldsymbol{v}\|_{U} \\
\lesssim & \left\|\boldsymbol{g}_{u}\right\|_{H^{\frac{1}{2}\left(\partial_{u} \Omega\right)}}\|\tau\|_{\Sigma}+\|\boldsymbol{f}\|_{\Omega}\|\boldsymbol{v}\|_{U} \cdot(5.33)
\end{aligned}
$$

The result now follows readily from this estimate, Theorems 1 and 2, and standard saddle point theory [8].

\section{Discretization}

In this section, we discretize the continuous problem (4.4) using conforming finite elements. The notion of conformity and the choice of mixed finite element spaces are discussed in Section 6.1 and the resulting discretized problem is presented and analyzed in Section 6.2.

\subsection{Discrete spaces}

For each $i \in I$, we introduce a shape-regular, simplicial grid $\Omega_{i, h}$ which tessellates $\Omega_{i}$. The union of meshes of a given dimension $d$ (with $0 \leq d \leq n$ ) is denoted by $\Omega_{h}^{d}=\cup_{i \in I^{d}} \Omega_{i, h}$ and we define $\Omega_{h}=\cup_{i \in I} \Omega_{i, h}$. We let the grid respect all lower-dimensional features and be matching across all interfaces. The tesselation of $\Gamma$ is thus given by $\Gamma_{h}=\Gamma \cap \partial \Omega_{h}$. Moreover, the trace mesh $\Gamma_{j, h}$ and $\Omega_{i, h}$ are equivalent for each $j \in \hat{J}_{i}$. The typical mesh size is denoted by $h$ and we use $h$ as a subscript to indicate the discretized counterpart of functions and function spaces.

With the aim of obtaining a stable and conforming method, we search for a discrete solution in subspaces of the function spaces defined in Section 4. We choose discrete function spaces $\left(\Sigma_{h}, U_{h}, R_{h}\right)$ on the grid $\Omega_{h}$ according to the following three conditions:

(S1) The finite element spaces are conforming, i.e.:

$$
\Sigma_{h} \subset \Sigma, \quad \boldsymbol{U}_{h} \subset \boldsymbol{U}, \quad R_{h} \subset R .
$$

(S2) $\quad \Sigma_{h}$ and $\boldsymbol{U}_{h}$ are such that for each $i \in I$ :

$\nabla \cdot \Sigma_{i, h} \subseteq \boldsymbol{U}_{i, h} \quad$ and $\left.\quad\left(\boldsymbol{n} \cdot \Sigma_{\hat{\jmath}, h}\right)\right|_{\Gamma_{j}}=\boldsymbol{U}_{i, h}, \quad \forall j \in \hat{J}_{i}$.

(S3) A mixed-dimensional, finite element space $W_{h}$ exists such that:

(a) $\mathfrak{D} \times W_{h} \subseteq \Sigma_{h}$

(b) $\left(\Xi W_{i, h}\right) \times R_{i, h}$ forms a stable pair for the two-dimensional Stokes problem for each $i \in$ $I^{2}$.

(S4) $\quad \Sigma_{i, h} \times \boldsymbol{U}_{i, h} \times R_{i, h}$ forms a stable triplet for threedimensional, mixed elasticity for each $i \in I^{3}$.

We provide an exemplary family of finite elements satisfying all four conditions. This choice is most concisely described using the notation of finite element exterior calculus [3]. A translation to more conventional nomenclature is 
provided afterwards, for convenience. Given a polynomial degree $k \geq 0$, let

$$
\begin{aligned}
\Sigma_{h} & =\prod_{d=1}^{n} \prod_{i \in I^{d}}\left(P_{k+n-d+1} \Lambda^{d-1}\left(\Omega_{i, h}\right)\right)^{n}, \\
\boldsymbol{U}_{h} & =\prod_{d=0}^{n} \prod_{i \in I^{d}}\left(P_{k+n-d} \Lambda^{d}\left(\Omega_{i, h}\right)\right)^{n}, \\
R_{h} & =\prod_{d=2}^{n} \prod_{i \in I^{d}}\left(P_{k+n-d} \Lambda^{d}\left(\Omega_{i, h}\right)\right)^{k_{d}} .
\end{aligned}
$$

In other words, for $n=3: \Sigma_{i, h}$ with $i \in I^{3}$ corresponds to three rows of Nedelec elements of the second kind $\left(N 2_{k+1}^{f}\right.$ [20]) with degrees of freedom on the faces. For $i \in I^{2}$, it is three rows of Brezzi-Douglas-Marini elements (BDM $M_{k+n-1}$ [11]). Finally $\Sigma_{i, h}$ with $i \in I^{1}$ is given by a triplet of continuous Lagrange elements $\left(P_{k+2}\right)$. The spaces $\boldsymbol{U}_{i, h}$ and $R_{i, h}$ are defined for $i \in I$ as three and $k_{d_{i}}$ rows, respectively, of discontinuous Lagrange elements $\left(P_{-\left(k+n-d_{i}\right)}\right)$.

In this case, the auxiliary space $W_{h}$ of (S3) is explicitly given by:

$W_{h}=\prod_{d=2}^{n} \prod_{i \in I^{d}}\left(P_{k+n-d+2}^{-} \Lambda^{d-2}\left(\Omega_{i, h}\right)\right)^{n}$.

For $n=3, W_{i, h}$ is thus given by three rows of (first kind) edge-based Nedelec element $\left(N 1_{k+2}^{e}\right)$ for $i \in I^{3}$ and by three instances of Lagrange elements $\left(P_{k+n}\right)$ for $i \in I^{2}$. The lowest-order choice in this family, i.e., with $k=0$, is presented in Table 1.

A reduced family of finite elements arises by noting that all stability conditions remain valid after the polynomial order of the trace onto $\Gamma$ is reduced by 1 . Table 2 presents the lowest order member of this family.

Properties (S1)-(S3) can be verified with the use of the presented tables. Finally, these families correspond to the

Table 1 The finite element spaces chosen for $n=2$ and $n=$ 3 of lowest order within the family (6.1). The negative orders in the subscript denote discontinuous Lagrange elements. On the zerodimensional manifolds, the polynomial order is redundant since any finite element space corresponds to point evaluation there

\begin{tabular}{lllll}
\hline$d$ & $W_{h}$ & $\Sigma_{h}$ & $\boldsymbol{U}_{h}$ & $R_{h}$ \\
\hline 2 & $\left(P_{2}\right)^{2}$ & $\left(B D M_{1}\right)^{2}$ & $\left(P_{0}\right)^{2}$ & $P_{0}$ \\
1 & & $\left(P_{2}\right)^{2}$ & $\left(P_{-1}\right)^{2}$ & \\
0 & & & $\left(P_{0}\right)^{2}$ & \\
3 & $\left(N 1_{2}^{e}\right)^{3}$ & $\left(N 2_{1}^{f}\right)^{3}$ & $\left(P_{0}\right)^{3}$ & $\left(P_{0}\right)^{3}$ \\
2 & $\left(P_{3}\right)^{3}$ & $\left(B D M_{2}\right)^{3}$ & $\left(P_{-1}\right)^{3}$ & $P_{-1}$ \\
1 & & $\left(P_{3}\right)^{3}$ & $\left(P_{-2}\right)^{3}$ & \\
0 & & & $\left(P_{0}\right)^{3}$ & \\
\hline
\end{tabular}

Table 2 The lowest-order finite element spaces of the reduced family for $n=2$ and $n=3$. The superscript minus indicates that the trace of the finite element space onto $\Gamma_{h}$ is reduced by one order

\begin{tabular}{lllll}
\hline$d$ & $W_{h}$ & $\Sigma_{h}$ & $U_{h}$ & $R_{h}$ \\
\hline 2 & $\left(P_{2}^{-}\right)^{2}$ & $\left(B D M_{1}^{-}\right)^{2}$ & $\left(P_{0}\right)^{2}$ & $P_{0}$ \\
1 & & $\left(P_{1}\right)^{2}$ & $\left(P_{0}\right)^{2}$ & \\
0 & & & $\left(P_{0}\right)^{2}$ & \\
3 & $\left(N 1_{2}^{e-}\right)^{3}$ & $\left(N 2_{1}^{-}\right)^{3}$ & $\left(P_{0}\right)^{3}$ & $\left(P_{0}\right)^{3}$ \\
2 & $\left(P_{2}^{-}\right)^{3}$ & $\left(B D M_{1}^{-}\right)^{3}$ & $\left(P_{0}\right)^{3}$ & $P_{0}$ \\
1 & & $\left(P_{1}\right)^{3}$ & $\left(P_{0}\right)^{3}$ & \\
0 & & & $\left(P_{0}\right)^{3}$ & \\
\hline
\end{tabular}

stable triplets analyzed in [3] (Sections 11.6-11.7); hence, (S4) holds as well.

\subsection{Discrete problem}

Since the finite elements described above are contained in the continuous spaces from Section 5 by (S1), the discrete formulation of the model problem is a direct translation of Eq. 4.4:

Find $\left(\sigma_{h}, \boldsymbol{u}_{h}, r_{h}\right) \in \Sigma_{h} \times \boldsymbol{U}_{h} \times R_{h}$ such that

$$
\begin{aligned}
\left(\mathfrak{A} \sigma_{h}, \tau_{h}\right)_{\Omega \times \Gamma}+\left(\boldsymbol{u}_{h}, \mathfrak{D} \cdot \tau_{h}\right)_{\Omega}+ & \left(r_{h}, \operatorname{skw} \tau_{h}\right)_{\Omega}=\left(\boldsymbol{g}_{u}, \boldsymbol{n} \cdot \tau_{h}\right)_{\partial_{u} \Omega}, \\
\left(\mathfrak{D} \cdot \sigma_{h}, \boldsymbol{v}_{h}\right)_{\Omega}=-\left(\boldsymbol{f}, \boldsymbol{v}_{h}\right)_{\Omega}, \quad(6.2 \mathrm{a}) &
\end{aligned}
$$$$
\left(\mathrm{skw} \sigma_{h}, s_{h}\right)_{\Omega}=0 \text {. }
$$

for all $\left(\tau_{h}, \boldsymbol{v}_{h}, s_{h}\right) \in \Sigma_{h} \times \boldsymbol{U}_{h} \times R_{h}$. We note that the saddlepoint structure of this problem has not changed, and can readily be uncovered using the bilinear forms from Eq. 4.5.

\subsection{Stability}

We continue with the analysis concerning the wellposedness of Eq. 6.2. Let us recall the norms from Eq. 5.1 for convenience:

$$
\begin{aligned}
\|\tau\|_{\Sigma}^{2} & :=\|\tau\|_{\Omega}^{2}+\|\boldsymbol{n} \cdot \tau\|_{\Gamma}^{2}+\|\mathfrak{D} \cdot \tau\|_{\Omega}^{2}, \\
\|\boldsymbol{v}\|_{U}^{2} & :=\|\boldsymbol{v}\|_{\Omega}^{2}, \\
\|s\|_{R}^{2} & :=\|s\|_{\Omega}^{2} .
\end{aligned}
$$

Theorem 4 (Ellipticity) If the discrete spaces satisfy (S2) and $\sigma_{h} \in \Sigma_{h}$ satisfies:

$b\left(\sigma_{h} ; \boldsymbol{v}_{h}, s_{h}\right)=0, \quad$ for all $\left(\boldsymbol{v}_{h}, s_{h}\right) \in \boldsymbol{U}_{h} \times R_{h}$,

then the following ellipticity bound holds:

$a\left(\sigma_{h} ; \sigma_{h}\right) \gtrsim\left\|\sigma_{h}\right\|_{\Sigma}^{2}$. 
Proof By (S2), we have $\mathfrak{D} \cdot \Sigma_{h} \subseteq \boldsymbol{U}_{h}$ and the same arguments are used as in Theorem 1.

The next step is to consider the inf-sup condition for the bilinear form $b$ in the discrete case.

Theorem 5 (Inf-Sup) If the discrete spaces satisfy conditions (S1)-(S4), then for all $\left(\boldsymbol{u}_{h}, r_{h}\right) \in \boldsymbol{U}_{h} \times R_{h}$,

$$
\sup _{\tau_{h} \in \Sigma_{h}} \frac{b\left(\tau_{h} ; \boldsymbol{u}_{h}, r_{h}\right)}{\left\|\tau_{h}\right\|} \gtrsim\left\|\boldsymbol{u}_{h}\right\|_{U}+\left\|r_{h}\right\|_{R} \text {. }
$$

Proof With $\boldsymbol{u}_{h} \in \boldsymbol{U}_{h}$ and $r_{h} \in R_{h}$ given, we follow a similar strategy as in the proof of Theorem 2 . Here, we use Lemma 5 to construct $\eta_{h} \in \Sigma_{h}$ in order to gain control of $\boldsymbol{u}_{h}$ and use Lemma 6 to define a divergence-free function $\xi_{h} \in \Sigma_{h}$ that controls $r_{h} \in R_{h}$.

In short, we choose $\eta_{h}, \xi_{h} \in \Sigma_{h}$ such that

$\mathfrak{D} \cdot \eta_{h}=\boldsymbol{u}_{h}$,

$\mathfrak{D} \cdot \xi_{h}=0, \quad \Pi_{R_{h}} \mathrm{skw} \xi_{h}=r_{h}-\Pi_{R_{h}} \mathrm{skw} \eta_{h}$

with $\Pi_{R_{h}}$ the $L^{2}$ projection onto $R_{h}$. Moreover, the pair satisfies:

$\left\|\eta_{h}\right\|_{\Sigma}+\left\|\xi_{h}\right\|_{\Sigma} \lesssim\left\|\boldsymbol{u}_{h}\right\|_{U}+\left\|r_{h}\right\|_{R}$

Following the same steps as in Theorem 2, we then set $\tau_{h}=\eta_{h}+\xi_{h}$ so that

$$
\begin{aligned}
b\left(\tau_{h} ; \boldsymbol{u}_{h}, r_{h}\right) & =\left(\mathfrak{D} \cdot \tau_{h}, \boldsymbol{u}_{h}\right)_{\Omega}+\left(\operatorname{skw} \tau_{h}, r_{h}\right)_{\Omega} \\
& =\left(\mathfrak{D} \cdot \eta_{h}, \boldsymbol{u}_{h}\right)_{\Omega}+\left(\operatorname{skw} \eta_{h}+\operatorname{skw} \xi_{h}, r_{h}\right)_{\Omega} \\
& =\left\|\boldsymbol{u}_{h}\right\|_{U}^{2}+\left\|r_{h}\right\|_{R}^{2} \\
\left\|\tau_{h}\right\|_{\Sigma} & \lesssim\left\|\eta_{h}\right\|_{\Sigma}+\left\|\xi_{h}\right\|_{\Sigma} \lesssim\left\|\boldsymbol{u}_{h}\right\|_{U}+\left\|r_{h}\right\|_{R} .
\end{aligned}
$$

The proof is concluded by combining the above.

Lemma 5 For each $\boldsymbol{u}_{h} \in \boldsymbol{U}_{h}$, a function $\eta_{h} \in \Sigma_{h}$ exists such that

$\mathfrak{D} \cdot \eta_{h}=\boldsymbol{u}_{h}, \quad\left\|\eta_{h}\right\|_{\Sigma} \lesssim\left\|\boldsymbol{u}_{h}\right\|_{U}$

Proof We use the same steps as in Lemma 2 to hierarchically construct $\eta_{h} \in \Sigma_{h}$. A concise exposition follows, starting with $d=0$. For each $i \in I^{d}$, we use (S2) to first construct a discrete $\boldsymbol{\phi}_{j, h}$ in the trace space $\left.\left(\boldsymbol{n} \cdot \Sigma_{h}\right)\right|_{\Gamma_{j}}$ for all $j \in \hat{J}_{i}$ such that:

$-\llbracket \boldsymbol{\phi}_{h} \rrbracket_{i}=\boldsymbol{u}_{i, h}-\nabla \cdot \eta_{i, h}$,

$\left\|\boldsymbol{\phi}_{h}\right\|_{\Gamma^{d}} \lesssim\left\|\boldsymbol{u}_{h}\right\|_{\Omega^{d}}+\left\|\boldsymbol{\phi}_{h}\right\|_{\Gamma^{d-1}}$

in which $\nabla \cdot \eta_{i, h}$ and $\boldsymbol{\phi}_{j, h}$ are understood as zero for $i \in I^{0}$, $j \in \check{J}_{i}$. For $i \in I^{d+1}$, the function $\eta_{i, h}$ is then defined as the bounded, discrete $H\left(\operatorname{div}, \Omega_{i, h}\right)$-extension from [23] (Section 4.1.2). These steps are repeated by incrementing $d$ until $\phi_{h}$ is completely defined on $\Gamma_{h}$. Finally, we solve a discrete Poisson problem for each $i \in I^{3}$, in analogy with Eq. 5.13, to complete $\eta_{h} \in \Sigma_{h}$. It follows by the same arguments as in Theorem 2 that the constructed $\eta_{h}$ satisfies (6.4).

Lemma 6 Given $r_{h} \in R_{h}$, a function $\xi_{h} \in \Sigma_{h}$ exists such that

$\mathfrak{D} \cdot \xi_{h}=0, \quad \Pi_{R_{h}} \operatorname{skw} \xi_{h}=r_{h}, \quad\left\|\xi_{h}\right\|_{\Sigma} \lesssim\left\|r_{h}\right\|_{R}$.

with $\Pi_{R_{h}}$ the $L^{2}$ projection onto $R_{h}$.

Proof In this proof, we make extensive use of the discrete space $W_{h}$ from stability requirement (S3). In particular, we will first introduce $w_{h} \in W_{h}$ such that $\mathfrak{D} \times w_{h}$ controls $r_{i, h}$ for $i \in I^{2}$. Then, a correction is introduced using (S4) in order to control $r_{i, h}$ for $i \in I^{3}$ as well. For brevity, we omit the subscript $h$ on all variables within this proof.

As in Lemma 4, we start with an interface function $\phi$ defined on the trace mesh $\Gamma_{h}^{2}$ which serves first as a source function and second as a boundary condition. We proceed according to the following four steps.

1. We consider functions on $\Gamma_{h}^{2}$ in the trace space of $\left.\left(W_{h}\right)\right|_{\Omega^{3}}$ that vanish at all intersections and extremities. Let us therefore introduce the function space $\Phi_{h}$ as

$$
\Phi_{h}=\prod_{i \in I^{3}} \prod_{j \in \check{J}_{i}}\left\{\left.\phi \in\left(\boldsymbol{n}_{i} \times W_{i, h}\right)\right|_{\Gamma_{j}}:\left.\left(\boldsymbol{n}_{j} \cdot \phi\right)\right|_{\partial \Gamma_{j}}=0\right\} .
$$

It is important to note that the two instances of $\boldsymbol{n}$ in this definition are different. In particular, the former is defined as normal to $\partial \Omega_{i}$, of which $\Gamma_{j}$ is a subset, whereas the latter is normal with respect to the boundary $\partial \Gamma_{j}$.

We note that, since $\mathfrak{D} \times W_{h} \subseteq \Sigma_{h}$ by (S3), we have $W_{i, h} \subseteq\left(H\left(\operatorname{curl}, \Omega_{i}\right)\right)^{3}$ for $i \in I^{3}$. In turn, it follows in the discrete setting that $\Phi_{j, h} \in\left(H\left(\operatorname{div}, \Gamma_{j}\right)\right)^{3}$ for all $j \in \breve{J}_{i}$, with the divergence tangential to $\Gamma_{j}$. Using this observation, we let $\phi$ solve the following minimization problem:

$\min _{\varphi \in \Phi_{h}} \frac{1}{2}\|\varphi\|_{H\left(\operatorname{div}, \Gamma^{2}\right)}^{2}$

subject to

$\Pi_{\mathbb{R}_{i}}\left(\mathrm{skw}_{2} \llbracket \varphi \rrbracket_{i}+r_{i}\right)=0, \quad \forall i \in I^{2}$.

In other words, a finite-dimensional problem is solved for each $i \in I^{2}$ to obtain a bounded distribution 
that has an average asymmetry corresponding to $r_{i}$. In particular, we obtain the following two properties:

$$
\begin{aligned}
-\Pi_{\mathbb{R}_{i}} \mathrm{skw}_{2} \llbracket \phi \rrbracket_{i} & =\Pi_{\mathbb{R}_{i}} r_{i}, \quad \forall i \in I^{2}, \\
\|\phi\|_{H\left(\operatorname{div}, \Gamma^{2}\right)} & \lesssim\|r\|_{\Omega^{2}} .
\end{aligned}
$$

2. We generate $w_{i}$ for $i \in I^{2}$ using the distribution $\phi$ from the first step as a source term. Introducing $\Theta_{i, h}=$ $\Xi W_{i, h}$, it follows from (S3) that $\Theta_{i, h} \times R_{i, h}$ is a stable pair for the discretization of the Stokes problem (5.27a). Hence, we can find $\theta_{i} \in \Theta_{i, h}$ such that:

$$
\begin{aligned}
\Pi_{R_{i, h}} \nabla \cdot \theta_{i} & =\left(\Pi_{R_{i, h}}-\Pi_{\mathbb{R}_{i}}\right)\left(r_{i}+\mathrm{skw}_{2} \llbracket \phi \rrbracket\right), \\
\left.\left(\theta_{i}\right)\right|_{\partial \Omega_{i}} & =0, \\
\|\theta\|_{H^{1}\left(\Omega^{2}\right)} & \lesssim\|r\|_{\Omega^{2}}+\|\phi\|_{\Gamma^{2}} \lesssim\|r\|_{\Omega^{2}}
\end{aligned}
$$

with $\Pi_{R_{i, h}}$ the $L^{2}$ projection onto $R_{i, h}$. We set $w_{i}=$ $\Xi^{-1} \theta_{i} \in W_{i, h}$ for all $i \in I^{2}$.

3. For $i \in I^{3}$, let $w_{i}$ be given by any bounded extension of $\phi$ into $H$ (curl, $\Omega_{i}$ ), i.e., $w_{i}$ is chosen for all $i \in I^{3}$ such that

$$
\begin{aligned}
\left.\left(\boldsymbol{n} \times w_{i}\right)\right|_{\Gamma_{j}} & =\phi_{j}, \quad \forall j \in \check{J}_{i} \\
\|w\|_{H\left(\operatorname{curl}, \Omega^{3}\right)} & \lesssim\|\phi\|_{H\left(\operatorname{div}, \Gamma^{2}\right)}
\end{aligned}
$$

4. Finally, we gain control of $r_{i}$ for $i \in I^{3}$. We recall that by stability condition (S4), the spaces $\Sigma_{i, h} \times \boldsymbol{U}_{i, h} \times$ $R_{i, h}$ form a stable triplet for the mixed formulation of elasticity with relaxed symmetry. Considering $\Gamma^{2}$ as a zero-traction boundary condition, we use the inf-sup condition associated to this stability to form a function $\psi_{i} \in \Sigma_{i, h}$ such that:

$$
\begin{aligned}
\nabla \cdot \psi_{i} & =0 \\
\Pi_{R_{i, h}} \operatorname{skw}_{3} \psi_{i} & =r_{i}-\Pi_{R_{i, h}} \operatorname{skw}_{3}\left(\nabla \times w_{i}\right) \\
\left.\left(\boldsymbol{n} \cdot \psi_{i}\right)\right|_{\Gamma_{j}} & =0 \\
\|\psi\|_{H\left(\mathrm{div}, \Omega^{3}\right)} & \lesssim\|r\|_{\Omega^{3}}+\|w\|_{H\left(\mathrm{curl}, \Omega^{3}\right)}
\end{aligned}
$$

To complete the mixed-dimensional function $\psi \in \Sigma_{h}$, we set $\psi_{i}=0$ for $i \notin I^{3}$.

Using the above ingredients, we set $\xi=\psi+\mathfrak{D} \times w$ and obtain the first two desired properties:

$$
\begin{array}{rlrl}
\mathfrak{D} \cdot \xi & =\mathfrak{D} \cdot(\psi+\mathfrak{D} \times w)=0, & \\
\left.\left(\Pi_{R_{h}} \mathrm{skw} \xi\right)\right|_{\Omega_{i}} & =\Pi_{R_{i, h}}(\operatorname{skw} \mathfrak{D} \times w) & \\
& =\Pi_{R_{i, h}} \mathrm{skw}_{2}\left(\nabla^{\perp} w_{i}-\llbracket n \times w \rrbracket_{i}\right) & \\
& =\Pi_{R_{i, h}}\left(\nabla \cdot \theta_{i}-\operatorname{skw}_{2} \llbracket \phi \rrbracket_{i}\right)=r_{i}, & & \forall i \in I^{2}, \\
\left.\left(\Pi_{R_{h}} \mathrm{skw} \xi\right)\right|_{\Omega_{i}} & =\Pi_{R_{i, h}} \operatorname{skw}_{3}\left(\psi_{i}+\nabla \times w_{i}\right)=r_{i}, & & \forall i \in I^{3} .
\end{array}
$$

The bound now follows by the estimates given in each step:

$\|\xi\|_{\Sigma}=\|\xi\|_{\Omega}+\|\boldsymbol{n} \cdot \xi\|_{\Gamma} \lesssim\left\|r_{h}\right\|_{R}$
The two previous theorems provide the sufficient ingredients to show stability of the discretization, formally presented in the following theorem.

Theorem 6 (Stability) If the discrete spaces satisfy the conditions (S1)-(S4), then the resulting mixed finite element method is stable; i.e., a unique solution exists satisfying the bound:

$\left\|\sigma_{h}\right\|_{\Sigma}+\left\|\boldsymbol{u}_{h}\right\|_{U}+\left\|r_{h}\right\|_{R} \lesssim\|\boldsymbol{f}\|_{\Omega}+\left\|\boldsymbol{g}_{u}\right\|_{H^{\frac{1}{2}}\left(\partial_{u} \Omega\right)}$

with a constant independent of the grid size $h$.

Proof Using Theorems 4 and 5, this result follows from standard saddle point theory [8].

\subsection{Convergence}

By consistency of the discretized problem (6.2) with respect to the continuous formulation (4.4) and stability from Theorem 6, we have shown that the proposed mixed finite element discretization is convergent. In turn, this section is devoted to obtaining the rates of convergence through a priori error estimation.

Let $\Pi_{R_{h}}$ and $\Pi_{U_{h}}$ be the $L^{2}$-projection operators onto the finite element spaces $R_{h}$ and $\boldsymbol{U}_{h}$. Moreover, under the assumption of sufficient regularity, we introduce the canonical projection operator $\Pi_{\Sigma_{h}}$ onto $\Sigma_{h}$ with the following commutativity properties:

$$
\begin{aligned}
\Pi_{U_{i, h}} \nabla \cdot \sigma_{i} & =\nabla \cdot \Pi_{\Sigma_{i, h}} \sigma_{i}, \\
\left.\Pi_{U_{i, h}}\left(\boldsymbol{n} \cdot \sigma_{\hat{\jmath}}\right)\right|_{\Gamma_{j}} & =\left.\left(\boldsymbol{n} \cdot \Pi_{\Sigma_{\hat{\jmath}, h}} \sigma_{\hat{\jmath}}\right)\right|_{\Gamma_{j}}, \quad \forall i \in I, j \in \hat{J}_{i} .
\end{aligned}
$$

A direct consequence of these two properties is that, for sufficiently regular $\sigma \in \Sigma$, we have the commuting property:

$\Pi_{U_{h}} \mathfrak{D} \cdot \sigma=\mathfrak{D} \cdot \Pi_{\Sigma_{h}} \sigma$.

Using $\|\cdot\|_{\rho, \Omega}$ as short-hand notation for the $H^{\rho}(\Omega)$ norm, the projection operator $\Sigma_{h}$ has the following approximation properties for $0 \leq \rho \leq k_{i}+1$ :

$$
\left\|\left(I-\Pi_{\Sigma_{i, h}}\right) \sigma_{i}\right\|_{\Omega_{i}} \lesssim h^{\rho}\left\|\sigma_{i}\right\|_{\rho, \Omega_{i}}, \quad i \in \cup_{d=1}^{n} I^{d},
$$

The maximal rate is given by $k_{i}=(k+1)+\left(n-d_{i}\right)$ for the full spaces (see Table 1) and $k_{i}=k+1$ for the reduced spaces (Table 2). Additionally, we have the following properties for $0 \leq \rho \leq k_{i}$ :

$$
\begin{aligned}
\left\|\nabla \cdot\left(\left(I-\Pi_{\Sigma_{i, h}}\right) \sigma_{i}\right)\right\|_{\Omega_{i}} & \lesssim h^{\rho}\left\|\nabla \cdot \sigma_{i}\right\|_{\rho, \Omega_{i}}, & & i \in \cup_{d=1}^{n} I^{d}, \\
\left\|\boldsymbol{n} \cdot\left(\left(I-\Pi_{\sum_{\hat{j}, h}}\right) \sigma_{\hat{\jmath}}\right)\right\|_{\Gamma_{j}} & \lesssim h^{\rho}\left\|\boldsymbol{n} \cdot \sigma_{\hat{\jmath}}\right\|_{\rho, \Gamma_{j}}, & & i \in \cup_{d=0}^{n-1} I^{d}, j \in \hat{J}_{i},(6.16 \mathrm{~b}) \\
\left\|\left(I-\Pi_{U_{i, h}}\right) \boldsymbol{u}_{i}\right\|_{\Omega_{i}} & \lesssim h^{\rho}\left\|\boldsymbol{u}_{i}\right\|_{\rho, \Omega_{i}}, & & i \in I, \\
\left\|\left(I-\Pi_{R_{i, h}}\right) r_{i}\right\|_{\Omega_{i}} & \lesssim h^{\rho}\left\|r_{i}\right\|_{\rho, \Omega_{i}}, & & i \in \cup_{d=2}^{n} I^{d},
\end{aligned}
$$


Note that for $\Omega^{0}$ and $\Gamma^{0}$, the projection operators correspond to the identity operator which makes Eqs. $6.16 \mathrm{c}$ and $6.16 \mathrm{~d}$ trivial there.

Theorem 7 (Convergence) Given $(\sigma, \boldsymbol{u}, r)$ as the solution to Eq. 4.4. Under the assumption of sufficient regularity, then the scheme converges optimally, i.e., the finite element solution $\left(\sigma_{h}, \boldsymbol{u}_{h}, r_{h}\right)$ to Eq. 6.2 satisfies the following estimate:

$$
\begin{gathered}
\left\|\sigma-\sigma_{h}\right\|_{\Sigma}+\left\|\boldsymbol{u}-\boldsymbol{u}_{h}\right\|_{U}+\left\|r-r_{h}\right\|_{R} \lesssim \\
\sum_{i \in I} h^{k_{i}}\left(h\|\sigma\|_{k_{i}+1, \Omega_{i}}+\|\mathfrak{D} \cdot \sigma\|_{k_{i}, \Omega_{i}}+\sum_{j \in \hat{J}_{i}}\|\boldsymbol{n} \cdot \sigma\|_{k_{i}, \Gamma_{j}}\right. \\
\left.+\|\boldsymbol{u}\|_{k_{i}, \Omega_{i}}+\|r\|_{k_{i}, \Omega_{i}}\right)
\end{gathered}
$$

Proof We restrict our choice of test functions $\left(\tau_{h}, \boldsymbol{v}_{h}, s_{h}\right)$ to the finite element spaces and subtract the discrete (6.2) from the continuous (4.4). We then obtain:

$a\left(\sigma-\sigma_{h} ; \tau_{h}\right)+b\left(\tau_{h} ; \boldsymbol{u}-\boldsymbol{u}_{h}, r-r_{h}\right)-b\left(\sigma-\sigma_{h} ; \boldsymbol{v}_{h}, s_{h}\right)=0$.

The projection operators from Eq. 6.16 are then used to project the true solution onto the finite element spaces. Introducing $\sigma_{\Pi}:=\Pi_{\Sigma_{h}} \sigma, \boldsymbol{u}_{\Pi}:=\Pi_{U_{h}} \boldsymbol{u}$, and $r_{\Pi}:=\Pi_{R_{h}} r$, we rewrite the above equation to:

$$
\begin{aligned}
a\left(\sigma_{\Pi}-\sigma_{h} ; \tau_{h}\right)+ & b\left(\tau_{h} ; \boldsymbol{u}_{\Pi}-\boldsymbol{u}_{h}, r_{\Pi}-r_{h}\right)-b\left(\sigma_{\Pi}-\sigma_{h} ; \boldsymbol{v}_{h}, s_{h}\right) \\
= & a\left(\sigma_{\Pi}-\sigma ; \tau_{h}\right)+b\left(\tau_{h} ; \boldsymbol{u}_{\Pi}-\boldsymbol{u}, r_{\Pi}-r\right) \\
& -b\left(\sigma_{\Pi}-\sigma ; \boldsymbol{v}_{h}, s_{h}\right)
\end{aligned}
$$

Let $\tau_{b, h}$ be the discrete stress from the construction in Theorem 5 with the following properties:

$$
\begin{aligned}
b\left(\tau_{b, h} ; \boldsymbol{u}_{\Pi}-\boldsymbol{u}_{h}, r_{\Pi}-r_{h}\right) & =\left\|\boldsymbol{u}_{\Pi}-\boldsymbol{u}_{h}\right\|_{U}^{2}+\left\|r_{\Pi}-r_{h}\right\|_{R}^{2} \\
\left\|\tau_{b, h}\right\|_{\Sigma} & \lesssim\left\|\boldsymbol{u}_{\Pi}-\boldsymbol{u}_{h}\right\|_{U}+\left\|r_{\Pi}-r_{h}\right\|_{R}
\end{aligned}
$$

The discrete test functions are then chosen to be:

$$
\tau_{h}:=\sigma_{\Pi}-\sigma_{h}+\delta \tau_{b, h}, \quad \boldsymbol{v}_{h}:=\boldsymbol{u}_{\Pi}-\boldsymbol{u}_{h}, \quad s_{h}:=r_{\Pi}-r_{h},
$$

with $\delta>0$ a constant to be determined later. Substituting this choice of functions into the left-hand side of Eq. 6.17 gives us:

$$
\begin{aligned}
a\left(\sigma_{\Pi}-\sigma_{h} ; \tau_{h}\right)= & a\left(\sigma_{\Pi}-\sigma_{h} ; \sigma_{\Pi}-\sigma_{h}\right)+a\left(\sigma_{\Pi}-\sigma_{h} ; \delta \tau_{b, h}\right), \\
& b\left(\tau_{h} ; \boldsymbol{u}_{\Pi}-\boldsymbol{u}_{h}, r_{\Pi}-r_{h}\right) \\
& -b\left(\sigma_{\Pi}-\sigma_{h} ; \boldsymbol{v}_{h}, s_{h}\right) \\
= & b\left(\delta \tau_{b, h} ; \boldsymbol{u}_{\Pi}-\boldsymbol{u}_{h}, r_{\Pi}-r_{h}\right) \\
= & \delta\left(\left\|\boldsymbol{u}_{\Pi}-\boldsymbol{u}_{h}\right\|_{U}^{2}+\left\|r_{\Pi}-r_{h}\right\|_{R}^{2}\right) .
\end{aligned}
$$

Next, due to Eqs. 6.15 and 6.1 , we have $\mathfrak{D} \cdot\left(\sigma_{\Pi}-\sigma_{h}\right)=$ $\Pi_{U_{h}} \mathfrak{D} \cdot\left(\sigma-\sigma_{h}\right)=0$. Hence, the coercivity of $\mathfrak{A}$ from Eq. 4.2 allows us to bound $a\left(\sigma_{\Pi}-\sigma_{h} ; \sigma_{\Pi}-\sigma_{h}\right)$ from below by $\left\|\sigma_{\Pi}-\sigma_{h}\right\|_{\Sigma}^{2}$. We then obtain the following bound with respect to the right-hand side of Eq. 6.17:

$$
\begin{aligned}
\left\|\sigma_{\Pi}-\sigma_{h}\right\|_{\Sigma}^{2} & +\delta\left(\left\|\boldsymbol{u}_{\Pi}-\boldsymbol{u}_{h}\right\|_{U}^{2}+\left\|r_{\Pi}-r_{h}\right\|_{R}^{2}\right) \lesssim \\
& -a\left(\sigma_{\Pi}-\sigma_{h} ; \delta \tau_{b, h}\right)+a\left(\sigma_{\Pi}-\sigma ; \sigma_{\Pi}-\sigma_{h}+\delta \tau_{b, h}\right) \\
& +b\left(\sigma_{\Pi}-\sigma_{h}+\delta \tau_{b, h} ; \boldsymbol{u}_{\Pi}-\boldsymbol{u}, r_{\Pi}-r\right) \\
& -b\left(\sigma_{\Pi}-\sigma ; \boldsymbol{u}_{\Pi}-\boldsymbol{u}_{h}, r_{\Pi}-r_{h}\right) .
\end{aligned}
$$

Next, we use the continuity of the forms $a$ and $b$ from Lemma 1 to bound the right-hand side further:

$$
\begin{aligned}
\ldots \lesssim & \left\|\sigma_{\Pi}-\sigma_{h}\right\|_{\Sigma} \delta\left\|\tau_{b, h}\right\|_{\Sigma} \\
& +\left(\left\|\sigma_{\Pi}-\sigma_{h}\right\|_{\Sigma}+\delta\left\|\tau_{b, h}\right\|_{\Sigma}\right)\left(\left\|\sigma_{\Pi}-\sigma\right\|_{\Sigma}+\| \boldsymbol{u}_{\Pi}\right. \\
& \left.-\boldsymbol{u}\left\|_{U}+\right\| r_{\Pi}-r \|_{R}\right) \\
& +\left(\left\|\boldsymbol{u}_{\Pi}-\boldsymbol{u}_{h}\right\|_{U}+\left\|r_{\Pi}-r_{h}\right\|_{R}\right)\left\|\sigma_{\Pi}-\sigma\right\|_{\Sigma} .
\end{aligned}
$$

An application of Young's inequality and rearranging terms then gives:

$$
\begin{aligned}
\left\|\sigma_{\Pi}-\sigma_{h}\right\|_{\Sigma}^{2}+ & \delta\left(\left\|\boldsymbol{u}_{\Pi}-\boldsymbol{u}_{h}\right\|_{U}^{2}+\left\|r_{\Pi}-r_{h}\right\|_{R}^{2}\right) \\
\lesssim & \delta^{2}\left\|\tau_{b, h}\right\|_{\Sigma}^{2}+\left(1+\delta^{-1}\right)\left\|\sigma_{\Pi}-\sigma\right\|_{\Sigma}^{2}+\| \boldsymbol{u}_{\Pi} \\
& -\boldsymbol{u}\left\|_{U}^{2}+\right\| r_{\Pi}-r \|_{R}^{2}
\end{aligned}
$$

Using Eq. $6.18 \mathrm{~b}$ and setting $\delta$ sufficiently small, depending only on the hidden constant in $\lesssim$, leads us to:

$$
\begin{aligned}
\left\|\sigma_{\Pi}-\sigma_{h}\right\|_{\Sigma}^{2} & +\left\|\boldsymbol{u}_{\Pi}-\boldsymbol{u}_{h}\right\|_{U}^{2}+\left\|r_{\Pi}-r_{h}\right\|_{R}^{2} \\
& \lesssim\left\|\sigma_{\Pi}-\sigma\right\|_{\Sigma}^{2}+\left\|\boldsymbol{u}_{\Pi}-\boldsymbol{u}\right\|_{U}^{2}+\left\|r_{\Pi}-r\right\|_{R}^{2}
\end{aligned}
$$

With the triangle inequality, we thus obtain the estimate:

$$
\begin{aligned}
\left\|\sigma-\sigma_{h}\right\|_{\Sigma} & +\left\|\boldsymbol{u}-\boldsymbol{u}_{h}\right\|_{U}+\left\|r-r_{h}\right\|_{R} \\
& \lesssim\left\|\sigma_{\Pi}-\sigma\right\|_{\Sigma}+\left\|\boldsymbol{u}_{\Pi}-\boldsymbol{u}\right\|_{U}+\left\|r_{\Pi}-r\right\|_{R} .
\end{aligned}
$$

An application of the approximation properties (6.16) then finishes the proof.

\section{Numerical experiments}

In this section, we aim to verify the analysis from the previous sections through numerical experiments. We consider two test cases in 2D, simulating stiff and flexible inclusions, respectively.

Both test cases are set on the unit square $(0,1)^{2}$ and we use the coupling conditions described by example 1 from Section 3.3. The thickness of each inclusion is given by $2 \epsilon=0.002$ and we set both Lamé parameters to unity in the two-dimensional bulk, for simplicity. 
The body force $\boldsymbol{f}$ is set to 0 and we impose the following, parabolic displacement field on the boundary of the domain:

$u(x, y)=\left[\begin{array}{l}0.01 y(1-y)(2 x-1) \\ 0.01 x(1-x)(2 y-1)\end{array}\right], \quad$ on $\partial_{u} \Omega$.

We note that since all boundary displacements are outward, the material will be in tension.

For the computations, we employ the reduced finite elements of lowest order given by Table 2 with $n=2$. The solution is computed on six refinement levels with the mesh size decreasing by approximately a half per level. The result from the finest mesh serves as the reference solution. Finally, we compute the error in each dimension with respect to this reference solution using the norms defined in Eq. 5.1.

The proof of convergence in Theorem 7 assumes sufficient regularity of the solution in order to guarantee firstorder convergence. This can generally not be guaranteed in the vicinity of intersection points. We therefore exclude a ball of radius 0.02 around each intersection point from the error calculation.

The code, implemented using Python, is based on the mixed-dimensional structure of Porepy [17] combined with the finite elements from the FEniCS project [1]. All grids are conforming with respect to the inclusions and are generated using GMSH [16].

The differences between the test cases and the corresponding results are discussed in more detail in the following subsections.

\subsection{Test case: stiff inclusions}

The first test case considers a network of lower-dimensional inclusions in a pavement pattern, inspired by [24] and illustrated in Fig. 3 (left). On this geometry, we simulate stiff inclusions by increasing the shear moduli to $\mu_{\|}=\mu_{\perp}=$ 100 , i.e., a factor 100 larger than the shear modulus of the surrounding bulk.

The displacement field (not shown) is a smooth continuous field since the stiff inclusions effectively keep all the subdomains together. On the other hand, the stresses exhibit discontinuities since the inclusions can easily distribute the momentum in the tangential directions. These discontinuities are apparent in the volumetric stress field shown in Fig. 2 (right).

The irregular behavior of the stress field around the intersection points can clearly be observed. Qualitatively, the highest volumetric stress values can be seen at the right boundary of the domain, where the medium is stretched between an inclusion and the prescribed displacement at the boundary.

We continue with the results of the convergence test, presented in Table 3. Here, the first column represents the dimension of the variable and the second column is the mean mesh size in the two-dimensional bulk domain.

The overall convergence is of first order, thus in agreement with Theorem 7 . We observe that the network of inclusions dominates the system and effectively forms the boundary conditions for the two-dimensional domains, thus creating a one-way influence. This is reflected in the rates since we observe second-order convergence in the onedimensional stress variable, as can be expected from linear Lagrange elements in a one-dimensional problem. If the influence was more mutual, the first-order convergence of the bulk would pollute this rate, as we shall see in case 2 . On the other hand, the quadratic convergence is inherited at the intersection points, as shown in the bottom row of the table.

An outlier is the stress in the two-dimensional surroundings, showing a suboptimal rate of approximately 0.8-0.9. A possible explanation for this may lie in the comparison of the stress variable to the specified reference solution. In particular, a numerical integration between the non-matching
Fig. 2 The domain of computation for case 1 (left) and the obtained volumetric stress field (right). Due to the high rigidity of the inclusions, the stress field becomes discontinuous
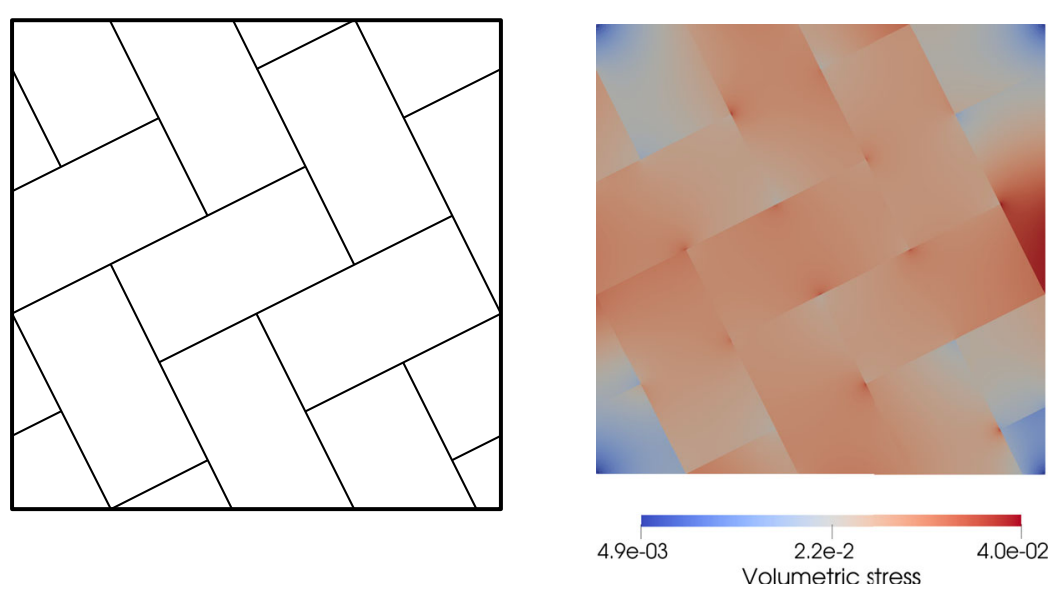
Table 3 Convergence results for case 1 . Convergence of at least first order can be observed in almost all variables, in accordance with the theory

\begin{tabular}{|c|c|c|c|c|c|c|c|}
\hline \multirow[t]{2}{*}{$d$} & \multirow[t]{2}{*}{$h$} & \multicolumn{2}{|l|}{$u$} & \multicolumn{2}{|l|}{$\sigma$} & \multicolumn{2}{|l|}{$r$} \\
\hline & & Error & Rate & Error & Rate & Error & Rate \\
\hline \multirow[t]{5}{*}{2} & $8.41 \mathrm{E}-02$ & $1.19 \mathrm{E}-04$ & & $1.04 \mathrm{E}-03$ & & $2.11 \mathrm{E}-04$ & \\
\hline & $5.34 \mathrm{E}-02$ & $7.66 \mathrm{E}-05$ & 0.97 & $6.86 \mathrm{E}-04$ & 0.92 & $1.36 \mathrm{E}-04$ & 0.98 \\
\hline & $2.71 \mathrm{E}-02$ & $4.06 \mathrm{E}-05$ & 0.94 & $3.91 \mathrm{E}-04$ & 0.83 & $7.58 \mathrm{E}-05$ & 0.86 \\
\hline & $1.43 \mathrm{E}-02$ & $2.15 \mathrm{E}-05$ & 1.00 & $2.22 \mathrm{E}-04$ & 0.89 & $4.23 \mathrm{E}-05$ & 0.92 \\
\hline & $7.44 \mathrm{E}-03$ & $1.11 \mathrm{E}-05$ & 1.01 & $1.26 \mathrm{E}-04$ & 0.86 & $2.28 \mathrm{E}-05$ & 0.94 \\
\hline \multirow[t]{5}{*}{1} & $8.41 \mathrm{E}-02$ & $5.02 \mathrm{E}-04$ & & $3.49 \mathrm{E}-03$ & & & \\
\hline & $5.34 \mathrm{E}-02$ & $1.72 \mathrm{E}-04$ & 2.37 & $2.33 \mathrm{E}-05$ & 11.05 & & \\
\hline & $2.71 \mathrm{E}-02$ & $9.02 \mathrm{E}-05$ & 0.95 & $5.05 \mathrm{E}-06$ & 2.25 & & \\
\hline & $1.43 \mathrm{E}-02$ & $4.64 \mathrm{E}-05$ & 1.04 & $8.92 \mathrm{E}-07$ & 2.72 & & \\
\hline & 7.44E-03 & $2.16 \mathrm{E}-05$ & 1.16 & $2.36 \mathrm{E}-07$ & 2.03 & & \\
\hline \multirow[t]{5}{*}{0} & 8.41E-02 & $1.49 \mathrm{E}-05$ & & & & & \\
\hline & $5.34 \mathrm{E}-02$ & $3.45 \mathrm{E}-06$ & 3.22 & & & & \\
\hline & $2.71 \mathrm{E}-02$ & $7.24 \mathrm{E}-07$ & 2.30 & & & & \\
\hline & $1.43 \mathrm{E}-02$ & $1.57 \mathrm{E}-07$ & 2.40 & & & & \\
\hline & 7.44E-03 & $2.79 \mathrm{E}-08$ & 2.63 & & & & \\
\hline
\end{tabular}

coarse and fine grids was used that may introduce additional, low-order errors. Another possibility is that the stress solution does not have sufficient regularity in the considered domain to allow for higher convergence rates.

Finally, the exceptional convergence of the stress in the inclusions at the first refinement is likely due to the large mesh size of the coarsest mesh. The rates are more in line with expectations at later refinements.

\subsection{Test case: flexible inclusions}

The second test case uses a geometry from the benchmarking study [15], as illustrated in Fig. 3 (left). Due to its connection to modeling fractures in porous media, we use this geometry to simulate flexible inclusions. The rigidity of the features is reduced by setting the shear moduli $\mu_{\|}=\mu_{\perp}=0.01$.

Due to the low rigidity, we expect the displacement field to become discontinuous across each inclusion. These discontinuities are indeed observed, as shown in Fig. 3 (right). As illustrated there, the deformation is several orders of magnitude smaller in the central square compared to the surrounding subdomains. This is to be expected since it is only held in place by its neighbors through the effect of the intermediate inclusions.

The convergence results for this test case are reported in Table 4. As can be seen from the rates, a majority of the variables exhibit linear convergence in all domains in
Fig. 3 The domain of computation for case 1 (left) and the obtained displacement field (right). Due to the low rigidity of the inclusions, the displacement field becomes discontinuous

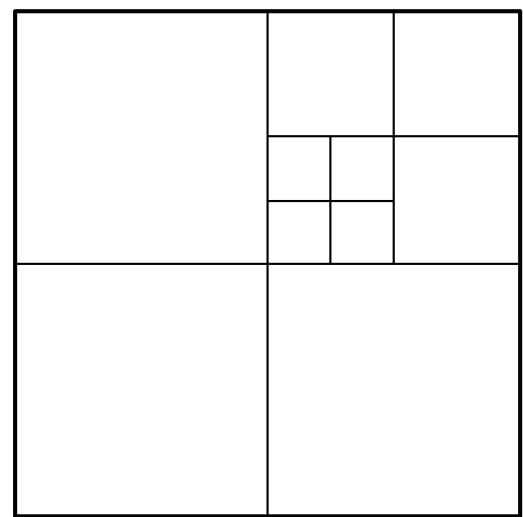


Table 4 Convergence results for Case 2. Overall, a more sporadic convergence behavior is observed compared to case 1

\begin{tabular}{|c|c|c|c|c|c|c|c|}
\hline \multirow[t]{2}{*}{$d$} & \multirow[t]{2}{*}{$h$} & \multicolumn{2}{|l|}{$u$} & \multicolumn{2}{|l|}{$\sigma$} & \multicolumn{2}{|l|}{$r$} \\
\hline & & Error & Rate & Error & Rate & Error & Rate \\
\hline \multirow[t]{5}{*}{2} & $1.22 \mathrm{E}-01$ & $1.76 \mathrm{E}-04$ & & $1.79 \mathrm{E}-03$ & & $3.05 \mathrm{E}-04$ & \\
\hline & $6.41 \mathrm{E}-02$ & $9.34 \mathrm{E}-05$ & 0.99 & $1.07 \mathrm{E}-03$ & 0.80 & $1.99 \mathrm{E}-04$ & 0.67 \\
\hline & $3.23 \mathrm{E}-02$ & $4.74 \mathrm{E}-05$ & 0.99 & $6.03 \mathrm{E}-04$ & 0.84 & $1.20 \mathrm{E}-04$ & 0.74 \\
\hline & $1.67 \mathrm{E}-02$ & $2.47 \mathrm{E}-05$ & 0.98 & $3.37 \mathrm{E}-04$ & 0.88 & $6.64 \mathrm{E}-05$ & 0.89 \\
\hline & $8.40 \mathrm{E}-03$ & $1.28 \mathrm{E}-05$ & 0.96 & $1.98 \mathrm{E}-04$ & 0.78 & $3.52 \mathrm{E}-05$ & 0.93 \\
\hline \multirow[t]{5}{*}{1} & $1.22 \mathrm{E}-01$ & $2.10 \mathrm{E}-04$ & & $4.91 \mathrm{E}-07$ & & & \\
\hline & $6.41 \mathrm{E}-02$ & $1.11 \mathrm{E}-04$ & 1.00 & $2.07 \mathrm{E}-07$ & 1.35 & & \\
\hline & $3.23 \mathrm{E}-02$ & $5.75 \mathrm{E}-05$ & 0.96 & $1.85 \mathrm{E}-07$ & 0.16 & & \\
\hline & $1.67 \mathrm{E}-02$ & $2.78 \mathrm{E}-05$ & 1.09 & $7.96 \mathrm{E}-08$ & 1.27 & & \\
\hline & $8.40 \mathrm{E}-03$ & $1.26 \mathrm{E}-05$ & 1.16 & $1.54 \mathrm{E}-08$ & 2.41 & & \\
\hline \multirow[t]{5}{*}{0} & $1.22 \mathrm{E}-01$ & $7.58 \mathrm{E}-04$ & & & & & \\
\hline & $6.41 \mathrm{E}-02$ & $5.23 \mathrm{E}-05$ & 4.18 & & & & \\
\hline & $3.23 \mathrm{E}-02$ & $2.02 \mathrm{E}-05$ & 1.39 & & & & \\
\hline & $1.67 \mathrm{E}-02$ & $1.20 \mathrm{E}-05$ & 0.78 & & & & \\
\hline & $8.40 \mathrm{E}-03$ & $4.72 \mathrm{E}-06$ & 1.37 & & & & \\
\hline
\end{tabular}

accordance with the theory from Section 6.4. However, the rates are more sporadic in this test case since the inclusions do not dominate the system in the same way as in case 1 .

We note that the displacement in the zero-dimensional intersection points is less well-behaved than the other variables. This is possibly due to the fact that all values can be represented exactly here and therefore, there is no approximation error. In turn, the error in this variable is entirely dependent on the error of the variables in the higherdimensional domains. Due to the soft inclusions considered in this test case, small errors in the stress field have a strong influence on the displacement of these points, which is reflected in these results.

Funding Open access funding provided by Royal Institute of Technology. The research of the authors was funded in part by the Norwegian Research Council grants 233736, 250223. The first author received from the German Research Foundation (DFG) supporting for this work through funding SFB 1313, Project Number 327154368.

Open Access This article is licensed under a Creative Commons Attribution 4.0 International License, which permits use, sharing, adaptation, distribution and reproduction in any medium or format, as long as you give appropriate credit to the original author(s) and the source, provide a link to the Creative Commons licence, and indicate if changes were made. The images or other third party material in this article are included in the article's Creative Commons licence, unless indicated otherwise in a credit line to the material. If material is not included in the article's Creative Commons licence and your intended use is not permitted by statutory regulation or exceeds the permitted use, you will need to obtain permission directly from the copyright holder. To view a copy of this licence, visit http:// creativecommonshorg/licenses/by/4.0/.

\section{References}

1. Alnæs, M., Blechta, J., Hake, J., Johansson, A., Kehlet, B., Logg, A., Richardson, C., Ring, J., Rognes, M.E., Wells, G.N.: The fenics project version 1.5. Archive of Numerical Software 3(100) (2015)

2. Arnold, D.N., Falk, R.S., Winther, R.: Differential complexes and stability of finite element methods II: the elasticity complex. IMA Volumes Math. Appl. 142, 47 (2006)

3. Arnold, D.N., Falk, R.S., Winther, R.: Finite element exterior calculus, homological techniques, and applications. Acta Numerica 15, 1-155 (2006)

4. Arnold, D.N., Winther, R.: Mixed finite elements for elasticity. Numer. Math. 92(3), 401-419 (2002)

5. Awanou, G.: Rectangular mixed elements for elasticity with weakly imposed symmetry condition. Adv. Comput. Math. 38(2), 351-367 (2013)

6. Bjørnarå, T.I.: Model development for efficient simulation of $\mathrm{co} 2$ storage. Ph.D. thesis, The University of Bergen (2018)

7. Bjørnarå, T.I., Nordbotten, J.M., Park, J.: Vertically integrated models for coupled two-phase flow and geomechanics in porous media. Water Resour. Res. 52(2), 1398-1417 (2016)

8. Boffi, D., Fortin, M., Brezzi, F.: Mixed Finite Element Methods and Applications. Springer Series in Computational Mathematics. Springer, Berlin (2013)

9. Boon, W.M., Nordbotten, J.M., Vatne, J.E.: Functional analysis and exterior calculus on mixed-dimensional geometries. Annali di Matematica Pura ed Applicata (1923-) (2020)

10. Boon, W.M., Nordbotten, J.M., Yotov, I.: Robust discretization of flow in fractured porous media. SIAM J. Numer. Anal. 56(4), 2203-2233 (2018)

11. Brezzi, F., Douglas, J., Marini, L.D.: Two families of mixed finite elements for second order elliptic problems. Numer. Math. 47(2), 217-235 (1985)

12. Caillerie, D., Nedelec, J.: The effect of a thin inclusion of high rigidity in an elastic body. Math. Meth. Appl. Sci. 2(3), 251-270 (1980) 
13. Ciarlet, P.: Mathematical Elasticity, Vol III, Theory of Shells. Mathematical Elasticity. Elsevier Science (2000)

14. Evans, L.: Partial Differential Equations. Orient Longman (1998)

15. Flemisch, B., Berre, I., Boon, W., Fumagalli, A., Schwenck, N., Scotti, A., Stefansson, I., Tatomir, A.: Benchmarks for singlephase flow in fractured porous media. Adv. Water Resour. 111, 239-258 (2018)

16. Geuzaine, C., Remacle, J.F.: Gmsh: a 3-d finite element mesh generator with built-in pre-and post-processing facilities. Int. J. Numer. Meth. Eng. 79(11), 1309-1331 (2009)

17. Keilegavlen, E., Berge, R., Fumagalli, A., Starnoni, M., Stefansson, I., Varela, J., Berre, I.: Porepy: an open-source software for simulation of multiphysics processes in fractured porous media. arXiv:1908.09869 (2019)

18. Licht, M.W.: Complexes of discrete distributional differential forms and their homology theory. Foundations of Computational Mathematics (2016)

19. Martin, V., Jaffré, J., Roberts, J.E.: Modeling fractures and barriers as interfaces for flow in porous media. SIAM J. Sci. Comput. 26(5), 1667-1691 (2005)
20. Nedelec, J.: Mixed finite elements in $\mathbb{R}^{3}$. Numer. Math. 35(3), 315-341 (1980)

21. Nordbotten, J.M., Boon, W.M.: Modeling, structure and discretization of mixed-dimensional partial differential equations. In: Domain Decomposition Methods in Science and Engineering XXIV, Lecture Notes in Computational Science and Engineering (2017)

22. Nordbotten, J.M., Celia, M.A.: Geological Storage of CO2: Modeling Approaches for Large-Scale Simulation. Wiley, New York (2011)

23. Quarteroni, A., Valli, A.: Domain Decomposition Methods for Partial Differential Equations. Oxford University Press, New York (1999)

24. Sævik, P., Nixon, C.: Inclusion of topological measurements into analytic estimates of effective permeability in fractured media. Water Resour. Res. 53(11), 9424-9443 (2017)

Publisher's note Springer Nature remains neutral with regard to jurisdictional claims in published maps and institutional affiliations. 